\title{
METTL3 regulates heterochromatin in mouse embryonic stem cells
}

Wenqi $\mathrm{Xu}^{1,2}$, Jiahui $\mathrm{Li}^{1}$, Chenxi $\mathrm{He}^{1}$, Jing Wen ${ }^{1}$, Honghui Ma ${ }^{1}$, Bowen Rong ${ }^{1}$, Jianbo Diao ${ }^{1}$, Liyong Wang ${ }^{1}$, Jiahua Wang ${ }^{1}$, Feizhen $\mathrm{Wu}^{1}$, Li Tan ${ }^{1}$, Yujiang Geno Shi ${ }^{3}$, Yang Shi ${ }^{4}$, , Hongjie Shen ${ }^{1, *}$

${ }^{1}$ Center for Medical Research and Innovation, Shanghai Pudong Hospital, Fudan University Pudong Medical Center, and the Shanghai Key Laboratory of Medical Epigenetics, the International Co-laboratory of Medical Epigenetics and Metabolism, Ministry of Science and Technology, Institutes of Biomedical Sciences, Fudan University, 2800 Gongwei Road, Pudong, Shanghai 201399, China.

${ }^{2}$ Key Laboratory of Carcinogenesis and Cancer Invasion, Ministry of Education, Liver Cancer Institute, Zhongshan Hospital, Fudan University, Shanghai, 200032, China.

${ }^{3}$ Division of Endocrinology, Diabetes and Hypertension, Department of Medicine, Brigham and Women's Hospital, Harvard Medical School, Boston, MA 02115, USA.

${ }^{4}$ Ludwig Institute for Cancer Research, Oxford Branch, Oxford University, UK

*Correspondence:

yang.shi@ludwig.ox.ac.uk (Y.S.)

hongjieshen@fudan.edu.cn (H.S.) 
METTL3 (methyltransferase-like 3) mediates mRNA $\mathbf{N}^{6}$-methyladenosine $\left(\mathrm{m}^{6} \mathbf{A}\right)$ methylation, which impacts mRNA stability and protein translation ${ }^{1}$. METTL3 has also been shown recently to bind chromatin ${ }^{2-4}$, but the role of METTL3 and $\mathbf{m}^{\mathbf{6}} \mathbf{A}$ methylation in the chromatin context is not fully understood. Here we report a direct role of METTL3 in regulating heterochromatin in mouse embryonic stem cells (mESCs), whose integrity is critical for silencing retroviral elements and for mammalian development ${ }^{5}$. We demonstrate that METTL3 predominantly localizes to the intracisternal A particles (IAPs)-type endogenous retroviruses family. Importantly, Mettl3 knockout impairs deposition of multiple heterochromatin marks onto METTL3-targeted IAPs, and upregulates IAP transcription, suggesting that METTL3 is important for IAP heterochromatin integrity. We provide further evidence that RNA transcripts derived from METTL3-bound IAP-types are associated with chromatin and are $\mathbf{m}^{6} \mathrm{~A}$ methylated. These $\mathbf{m}^{6} \mathrm{~A}$-marked transcripts are bound by the $\mathbf{m}^{6} \mathrm{~A}$ reader $\mathrm{YTHDC1}$, which interacts with METTL3 and in turns promotes METTL3 chromatin association. Additionally, METTL3 also physically interacts with the H3K9 tri-methyltransferase SETDB1 and cofactor TRIM28 and is important for their localization to IAPs. Taken together, our findings demonstrate that METTL3-catalysed RNA $\mathbf{m}^{6} \mathrm{~A}$ modification is important for IAP heterochromatin integrity in mESCs, thus revealing a novel mechanism of heterochromatin regulation in mammals.

\section{To understand the function and mechanism of action of METTL3 in chromatin regulation in} mESCs, we first interrogated METTL3 chromatin localizations and their association with 
47 heterochromatin and euchromatin histone marks, respectively. We found that out of a total of 1,928 METTL3 binding events (based on uniquely mapped reads only), a majority of them are associated with the two heterochromatin marks, H3K9me3 $(90.0 \%, 1,735 / 1,928)$ and H4K20me3 $(91.5 \%, 1,764 / 1,928)$, respectively, but METTL3 is rarely associated with the H3K27me3 repressive mark $(0.4 \%, 8 / 1,928)$ or the euchromatin mark, H3K4me3 $(5.1 \%$, 98/1,928). METTL3 has previously been reported to bind promoters of coding genes in cancer cell lines ${ }^{3,4}$. Consistently, we found a small percentage of METTL3 peaks mapped to promoters $(1.5 \%, 28 / 1,928)$ in $\mathrm{mESC}$, but this percentage increased significantly upon differentiation of mESCs to embryoid bodies (EBs) $(46.4 \%, 1,595 / 3,434)$. We further calculated the overlapping ratios (Jaccard statistics, see Methods) and relative distances between METTL3 binding sites and the peaks of histone modifications, both of which suggest a strong association between METTL3 and H3K9me3/H4K20me3 (Fig. 1a and Extended Data Fig. 1a). Their co-enrichment is further shown in the heatmap in Fig. 1b. These observations suggest that METTL3 may play a role at heterochromatin in $\mathrm{mESCs}$.

Heterochromatin establishment and maintenance are critical for gene regulation and genome integrity $^{6}$. In mammals, constitutive heterochromatin formed over repetitive elements including endogenous retroviruses (ERVs), such as IAP (intracisternal A-type particle), which are decorated by $\mathrm{H} 3 \mathrm{~K} 9 \mathrm{me} 3$ and $\mathrm{H} 4 \mathrm{~K} 20 \mathrm{me} 3^{7}$. $\mathrm{H} 3 \mathrm{~K} 9 \mathrm{me} 3$ is mediated by the methyltransferase SETDB1 (ESET or KMT1E) and its regulator TRIM28 (KAP1), both of which play a prominent role in IAP silencing in $\mathrm{mESCs}^{8,9}$. H4K20me3 is mediated by Suv4-20H1/2 and generally requires $\mathrm{H} 3 \mathrm{~K} 9 \mathrm{me} 3$ for its deposition ${ }^{10}$. METTL3 binding events are mainly enriched 
on ERVK elements (Extended Data Fig. 1b-c), which are decorated by H3K9me3 and H4K20me3, respectively (Extended Data Fig. 1d). A strong positive correlation between averaged METTL3 enrichment and those of H3K9me3 or H4K20me3 (Extended Data Fig. 1e) reveals co-enrichment of METTL3 and H3K9me3/H4K20me3 on specific subtypes of ERVK. Specifically, METTL3 appears to mostly bind the IAP elements, especially the IAPEz subtype, including the internal region of IAPEz (IAPEz-int) and the flanking cognate Long Terminal

Repeats (LTRs) (Fig. 1c). In addition to the intact IAPEz-int elements with lengths over $6 \mathrm{~Kb}$, many truncated IAPEz-int fragments are also defined as individual IAPEz-int elements in the Repeatmasker database, which might cause an over-representation of their total numbers. To reduce this bias, we stitched the adjacent IAPEz-int fragments and defined a total number of 2,542 IAPEz-int elements for further investigation (Extended Data Fig. 1f). We found METTL3 binding densities positively correlated with those of H3K9me3 (Fig. 1d) and H4K20me3 (Fig. 1e) on IAPEz-int elements. Furthermore, METTL3 is enriched throughout the entire body of IAPEz-int, similar to the distributions of H3K9me3 and H4K20me3, but with a stronger binding to the 5' end than the 3' end (Extended Data Fig. 1g and Fig. 1f). The specific binding of METTL3 to IAPEz, but not other retrotransposons was validated by ChIPqPCR using three different METTL3-specific antibodies (Extended Data Fig. 1h). Using either unique-only or unique+random mapping strategies (Extended Data Fig. 1i), we reached the same conclusion of co-enrichment of METTL3 with H3K9me3 and H4K20me3 over ERVKs in mESCs (Extended Data Fig. 1j). In order to display both site-specific and global features on repetitive elements, unique-only reads (for analyses on individual sites) and unique+random reads (for averaged analyses on repetitive elements classes) are both used in this study (see 
IAPEz-int is primarily controlled by $\mathrm{H} 3 \mathrm{~K} 9 \mathrm{me} 3$ in $\mathrm{mESCs}^{8,9}$. To investigate the functional significance of METTL3 enrichment over IAP elements, we first generated and validated Mettl3 KO and rescued cells containing either wildtype or catalytically compromised METTL3, METTL3 $^{\text {APPA }}$ (DPPW motif mutated to APPA) $)^{11}$ (Extended Data Fig. 2a-b). Importantly, we observed a significant decrease of the H3K9me3 and H4K20me3 levels on IAPEz-int elements in the Mettl3 KO cells, which was restored by METTL3 ${ }^{\mathrm{WT}}$, but not METTL3 ${ }^{\mathrm{APPA}}$ (Fig. 2a-b, and Extended Data Fig. 2c-e), suggesting that METTL3 regulates heterochromatin states on IAPEz-int via its catalytic activity. Consistently, depleting the $\mathrm{m}^{6} \mathrm{~A}$ demethylase, ALKBH5 ${ }^{12}$, led to a significant increase of H3K9me3 (Extended Data Fig. 2f-i). We found down-regulation of $\mathrm{H} 3 \mathrm{~K} 9 \mathrm{me} 3$ and $\mathrm{H} 4 \mathrm{~K} 20 \mathrm{me} 3$ upon Mettl3 KO is restricted to the METTL3-targeted IAPs, but not non-IAP ERVK or other types of repetitive elements, which are otherwise not bound by METTL3 (Extended Data Fig. 2j-k). Similarly, IAP-enriched histone variant H3. $3^{13}$ and DNA methylation ${ }^{14}$ important for transposon silencing were reduced upon Mettl3 KO, and were suggesting that METTL3 regulates DNA methylation via multiple mechanisms. Collectively, these findings suggest that METTL3 loss compromises heterochromatin integrity. 
By carrying out total RNA-seq, we observed a significant up-regulation of IAPEz-int transcription upon Mettl3 KO, which was suppressed by re-introduction of METTL3 ${ }^{\mathrm{WT}}$, but not METTL3 ${ }^{\text {APPA }}$ (Fig. 2c and Extended Data Fig. 3e). Furthermore, de-repression upon METTL3 loss is specific to IAPs (Extended Data Fig. 3f). The IAPEz-int RNA transcript level is negatively correlated with the densities of METTL3, H3K9me3 and H4K20me3 (Extended Data Fig. 3g-i) but not DNA methylation (Extended Data Fig. 3j), which does not play a dominant role in IAP silencing in $\mathrm{mESC}^{8}$. As METTL3 is known to regulate RNA stability ${ }^{15}$, we next determined and compared the stability of the transcript level of IAPEz-int with that of the coding gene, $N x t 1$, in the presence and absence of METTL3 at different time points post Actinomycin D treatment, which blocks transcription. As expected, degradation of the Nxt1 mRNA was severely impaired in the absence of METTL3 (Fig. 2d, Right), but the stability of the IAPEz-int RNAs remained unaltered (Fig. 2d, Left). Furthermore, re-analysis of the published RNA-seq data ${ }^{16}$ showed no increase of the IAP RNA levels in cells depleted of all three YTHDF proteins (YTHDF1/2/3) (Extended Data Fig. 3k), which are the $\mathrm{m}^{6} \mathrm{~A}$ readers reported to regulate RNA decay ${ }^{16,17}$. Taken together, these results suggest that METTL3mediated suppression of IAPEz-int is likely at the chromatin level.

In order to elucidate the mechanism by which METTL3 regulates IAPEz-int, we first compared METTL3 enrichment on METTL3-bound IAPEz-int elements in parental, Mettl3 KO and the two rescued cell lines. As expected, METTL3 enrichment on IAPEz-int was abolished in the Mettl3 KO cells and was restored by reintroducing METTL3 ${ }^{\mathrm{WT}}$. Surprisingly, METTL3 ${ }^{\mathrm{APPA}}$ and two additional METTL3 catalytic mutants, METTL3 ${ }^{\text {W475A }}$ and METTL3 ${ }^{\text {N477A }}$ (which 
disrupt the loop required for fencing catalytic cavity between METTL3 and METTL14) ${ }^{18}$ all

135

failed to localize to these repetitive elements, suggesting that association of METTL3 with chromatin may be dependent on its own catalytic activity (Fig. 3a-b, and Extended Data Fig. 4a-e). We also found depletion of METTL14 and other components of the METTL3/METTL14 methyltransferase complex reduced METTL3 chromatin association (Extended Data Fig. 4fk). In contrast, dCas9-guided, ectopically placed METTL3 ${ }^{\mathrm{WT}}$ or METTL3 ${ }^{\mathrm{APPA}}$ at IAPs in Mettl3 KO cells both induced H3K9me3 (Extended Data Fig. 41-n). This indicates that once successfully localized to a specific chromatin location, METTL3 has the ability to regulate H3K9me3 installment independent of its catalytic activity.

We next asked how METTL3 regulates H3K9me3 and heterochromatin formation. H3K9me3 at IAPEz-int is regulated by the SETDB1 methyltransferase and the associated factor TRIM28 $8^{8,9}$. Interestingly, TRIM28 was recently reported to interact with METTL3 ${ }^{19}$. Consistently, we found significant overlaps of the TRIM28 and SETDB1-bound IAPEz-int elements with those bound by METTL3 (Extended Data Fig. 5a) and positive correlations between their binding densities on these elements (Extended Data Fig. 5b). Furthermore, we found that METTL3 is co-immunoprecipitated with both SETDB1 and TRIM28 (Fig. 3c). Consequently, we next investigated whether METTL3 regulates the recruitment of SETDB1 and TRIM28 to IAPEz-int elements. We carried out ChIP-seq of SETDB1 and TRIM28 and found a decrease of both SETDB1 and TRIM28 in Mettl3 KO cells (Fig. 3d-e and Extended Data Fig. 5c-d), compared with the parental cells. Similar to H3K9me3, the decreased enrichment of SETDB1 is specific to IAP (Extended Data Fig. 5e). The decrease of TRIM28 
is most pronounced on IAPs, although it is also modestly reduced on other types of repetitive elements (Extended Data Fig. 5f). We found that catalytic mutation of METTL3 or inhibition of transcription by and large did not significantly affect the interactions of METTL3 with SETDB1 and TRIM28 (Extended Data Fig. 5g). These findings suggest that METTL3 facilitates the IAP-localization of SETDB1/TRIM28 to regulate H3K9me3 on chromatin, possibly through physical interactions. In contrast, the catalytic activity of METTL3 appears to be only necessary for METTL3 binding onto chromatin but not recruitment of the H3K9me3 methyltransferase machinery.

Given that the catalytic activity of METTL3 is important for METTL3's association with the IAPEz repetitive elements, we hypothesized that $\mathrm{m}^{6} \mathrm{~A}$ methylated transcripts at these locations may be important for METTL3 localization. Indeed, by fractionating whole cell extracts into proportion $(\sim 50 \%)$ of the IAPEz transcripts are associated with chromatin (Extended Data Fig. 6a). This finding is further confirmed by our ChIRP-seq (Extended Data Fig. 6b) and reanalysis of the published GRID-seq data ${ }^{20}$ (Extended Data Fig. 6c). We next carried out MeRIP using chromatin total RNA to detect $\mathrm{m}^{6} \mathrm{~A}$ on IAPEz transcripts and identified a total of 35,193 potential METTL3 dependent $\mathrm{m}^{6} \mathrm{~A}$ peaks genome-wide, which contain the classic DRACH consensus sequences, supporting the idea that they are bona fide $\mathrm{m}^{6} \mathrm{~A}$ peaks (Extended Data Fig. 6d). Consistent with the previous findings ${ }^{21,22}$, we showed that $\mathrm{m}^{6} \mathrm{~A}$ enrichment on the 3 ' ends of the mRNAs from coding genes is abrogated in the Mettl3 KO cells (Extended Data Fig. 
specifically enriched on their 5' and not 3' ends (Fig. 4a-b) (a total of $104 \mathrm{~m}^{6} \mathrm{~A}$ peaks localized to 86 IAPEz elements). The ability of MeRIP-seq to detect $\mathrm{m}^{6} \mathrm{~A}$-marked transcripts may be limited when transcripts are expressed at a low level such as the IAPEz transcripts, which can lead to under-estimation of the $\mathrm{m}^{6} \mathrm{~A}$ level. Indeed, as SETDB1 depletion increases IAPEz transcription $^{8}$, MeRIP-seq in the Setdb1 KO cells identified significantly increased $\mathrm{m}^{6}$ A signals on the 5 ' ends of IAPEz-int (a total of $752 \mathrm{~m}^{6} \mathrm{~A}$ peaks localized to 527 IAPEz elements) (Fig. 4c and Extended Data Fig. 6g-h). In order to rule out potential biases caused by the $\mathrm{m}^{6} \mathrm{~A}$ antibody used in the MeRIP experiments, we further validated $\mathrm{m}^{6} \mathrm{~A}$ modification on five adenosine sites residing at the 5' end of the IAPEz-int consensus sequence using SELECT, which is an elongation and ligation-based quantitative PCR amplification method for the detection of $\mathrm{m}^{6} \mathrm{~A}$ position at single-nucleoside resolution ${ }^{23}$ (Fig. 4d). The five adenosine sites identified by SELECT include the canonical site $\mathrm{GAA}^{159} \mathrm{CU}$, as well as non-canonical sites $\mathrm{UAA}^{107} \mathrm{AG}, \mathrm{UAA}^{132} \mathrm{GA}, \mathrm{GAA}^{135} \mathrm{GG}, \mathrm{GGA}{ }^{138} \mathrm{UU}$. Both the canonical (GAACU) 4 and one of the non-canonical sites, (GGAUU)4, can be methylated by METTL3/METTL14 in vitro, although to a less extent compared with the most optimal motif, (GGACU) 4 (Extended Data Fig. 6i).

RNA $\mathrm{m}^{6} \mathrm{~A}$ methylation is recognized by a family of proteins with a conserved YTH domain, which mediates recognition of $\mathrm{m}^{6} \mathrm{~A}^{1}$. We found that the nuclear $\mathrm{m}^{6} \mathrm{~A}$ reader, YTHDC $1^{24}$, is localized to IAPEz-int elements (Extended Data Fig. 7a). ChIP-seq showed a significant overlap between the binding sites of METTL3 and YTHDC1 (Extended Data Fig. 7b) and a significant positive correlation between their binding densities on IAPEz-int elements 

IAPEz-int. Supporting this hypothesis, we identified a significant reduction of YTHDC1's chromatin association in the Mettl3 KO cells, which was rescued by METTL3 ${ }^{\text {WT }}$, but not METTL3 $^{\text {APPA }}$ (Fig. 4e and Extended Data Fig. 7d-e), suggesting that YTHDC1 binding to chromatin is dependent on RNA $\mathrm{m}^{6} \mathrm{~A}$ methylation.

Given that YTHDC1 $\mathrm{KO}$ is cell lethal $\mathrm{l}^{25}$, to further investigate whether $\mathrm{m}^{6} \mathrm{~A}$ recognition is necessary for YTHDC1 binding to IAPEz, we generated three cell lines, all of which carry an integrated, AID-tagged YTHDC1, but differ on whether their endogenous Ythdcl is wildtype $\left(\mathrm{YTHDC} 1^{\mathrm{WT}}\right)$, a mutant for binding $\mathrm{m}^{6} \mathrm{~A}\left(\mathrm{YTHDC}^{\mathrm{W} 429 \mathrm{~A}}\right)^{24}$ or completely knocked out (Ythdc1 KO) (Extended Data Fig. 7f). These cells also express the Auxin receptor TIR1, thus the addition of the Auxin analog IAA can induce degradation of the AID-tagged YTHDC1 (Extended Data Fig. 7g). We found significant downregulation of YTHDC1 binding on IAPEz in the YTHDC1 $1^{\mathrm{W} 429 \mathrm{~A}}$ and Ythdcl KO cell lines (Extended Data Fig. 7h-k). Supporting these findings, inhibition of transcription using a number of different transcription inhibitors also led to a significant reduction of YTHDC1 binding (Extended Data Fig. 71).

217 Given that METTL3 recruitment requires its $\mathrm{m}^{6} \mathrm{~A}$ catalytic activity, we next asked whether YTHDC1 reciprocally contributes to METTL3 binding. Indeed, METTL3 binding is significantly impaired in the YTHDC1 ${ }^{\mathrm{W} 429 \mathrm{~A}}$ and the Ythdc1 $\mathrm{KO}$ cells (Fig. $4 \mathrm{f}$ and Extended 
Data Fig. 8g-j) on IAPEz-int. Furthermore, transcription from IAPEz-int is significantly

223 increased in both YTHDC1 $1^{\mathrm{W} 429 \mathrm{~A}}$ and Ythdc1 $\mathrm{KO}$ cells, consistent with an impaired heterochromatin in these cells (Fig. 4h). The reduction of H3K9me3 at IAPEz-int is not further exacerbated by depletion of YTHDC1 in the Mettl3 KO cells (Extended Data Fig. 8k-1), suggesting that METTL3 and YTHDC1 act in the same pathway to regulate heterochromatin on IAPEz-int elements. In addition, artificially tethering YTHDC1 to IAPEz by a CRISPRbased technology fails to restore H3K9me3 on these elements in Mettl3 KO cells (Extended

Data Fig. 8m-n), suggesting that YTHDC1 itself is not sufficient to induce heterochromatin formation. However, tethering YTHDC1 to IAPEz in the Mettl3 KO cells expressing the catalytic mutant, METTL3 $^{\mathrm{APPA}}$, induces a significant H3K9me3 increase on IAPEz (Extended Data Fig. 8o-p). Interestingly, we found METTL3 physically interacts with YTHDC1 (Fig. 4i), and this interaction is independent of transcription and the METTL3 catalytic activity 234 (Extended Data Fig. 8q), suggesting a potential biochemical mechanism for the observed 235 functional relationship between YTHDC1 and METTL3 in the recruitment of SETDB1/TRIM28 and regulation of IAP heterochromatin. Reciprocally, SETDB1 is also necessary for a stable association of METTL3 (Extended Data Fig. 9a-d) and YTHDC1

238 (Extended Data Fig. 9e-h) with IAPEz. Taken together, we propose that $\mathrm{m}^{6} \mathrm{~A}$ methylation by METTL3 provides a binding site for YTHDC1, which in turn recruits more METTL3 through physical interaction, forming a positive feedback loop that reinforces each other's localization to the IAPEz regions (Extended Data Fig. 10a). The IAPEz transcript-bound METTL3 recruits the H3K9 methyltransferase SETDB1/TRIM28 also through protein-protein interactions to 
install $\mathrm{H} 3 \mathrm{~K} 9$ trimethylation at these repetitive elements. The interactions of the METTL3 complex with SETDB1/TRIM28 similarly reinforce their respective occupancies at these genomic regions, revealing yet another layer of positive feedback loop, which ensures heterochromatin integrity. These positive feedback loops are reminiscent of the multiple feedback loops identified in S. pombe heterochromatin assembly ${ }^{26}$ (Extended Data Fig. 10b). Additionally, small RNAs are featured prominently in heterochromatin regulation in $S . p o m b e^{26}$, raising the question of whether small RNAs are also involved in IAPEz heterochromatin regulation. In this context, a recent study in mESCs suggested endo-siRNAs in repetitive element repression in response to the loss of DNA methylation ${ }^{27}$.

Lastly, H3K9 methylation at $S$. pombe meiotic genes, interestingly, involves not only the RNAidependent mechanism central to constitutive heterochromatin, but also a YTH domaincontaining protein $\mathrm{Mmi1}^{28}$ (Extended Data Fig. 10c). Mmil has been shown to interact with additional factors and link RNA recognition to H3K9 methylation and possibly the assembly of facultative heterochromatin at meiotic genes ${ }^{28}$. As discussed earlier, YTH domains in mammals have been shown to function as an RNA $\mathrm{m}^{6} \mathrm{~A}$ reader modality. However, the YTH domain in Mmi1 does not bind $\mathrm{m}^{6} \mathrm{~A}^{29}$ but rather recognizes the DSR (Determinant of Selective Removal) consensus motif on the RNAs transcribed from meiotic gene locus ${ }^{30}$, consistent with the fact that $S$. pombe lacks $\mathrm{m}^{6} \mathrm{~A}$ due to the absence of the $\mathrm{m}^{6} \mathrm{~A}$ enzyme, METTL3/METTL14 ${ }^{11}$. Importantly, we showed that the YTH domain in YTHDC1 is required for YTHDC1-mediated heterochromatin regulation on IAPEz-int in mESCs (Fig. 4 and Extended Data Fig. 8). Thus, the strategy of RNA recognition for $\mathrm{H} 3 \mathrm{~K} 9$ methylation at heterochromatic regions appears to 
be conserved in mammalian ESCs, although the exact molecular mechanisms are different.

Collectively, our findings identified a previously unappreciated yet crucial function of

268

269

270

271

272

273

274

275

276

277

278

279

280

281

282

283

284

285

286

287

288

289

290

291

292

293

294

295

296

297

298

299

300

301

302

METTL3 and $\mathrm{m}^{6} \mathrm{~A}$ methylation in heterochromatin regulation in mammalian embryonic stem

cells and revealed potential underlying mechanisms, thus providing important insight into not

only $\mathrm{m}^{6} \mathrm{~A}$ function but also mechanisms of heterochromatin regulation in mammals.

\section{References:}

1 Shi, H., Wei, J. \& He, C., Where, When, and How: Context-Dependent Functions of RNA Methylation Writers, Readers, and Erasers. MOL CELL 74640 (2019).

2 Knuckles, P. et al., RNA fate determination through cotranscriptional adenosine methylation and microprocessor binding. NAT STRUCT MOL BIOL 24561 (2017).

3 Barbieri, I. et al., Promoter-bound METTL3 maintains myeloid leukaemia by m(6)Adependent translation control. NATURE 552126 (2017).

4 Xiao, S. et al., The RNA N(6)-methyladenosine modification landscape of human fetal tissues. NAT CELL BIOL 21651 (2019).

5 Garcia-Perez, J. L., Widmann, T. J. \& Adams, I. R., The impact of transposable elements on mammalian development. DEVELOPMENT 1434101 (2016).

6 Allshire, R. C. \& Madhani, H. D., Ten principles of heterochromatin formation and function. Nat Rev Mol Cell Biol 19229 (2018).

7 Rowe, H. M. \& Trono, D., Dynamic control of endogenous retroviruses during development. VIROLOGY 411273 (2011).

8 Matsui, T. et al., Proviral silencing in embryonic stem cells requires the histone methyltransferase ESET. NATURE 464927 (2010).

9 Rowe, H. M. et al., KAP1 controls endogenous retroviruses in embryonic stem cells. NATURE 463237 (2010).

10 Schotta, G. et al., A silencing pathway to induce H3-K9 and H4-K20 trimethylation at constitutive heterochromatin. Genes Dev 181251 (2004).

11 Bujnicki, J. M., Feder, M., Radlinska, M. \& Blumenthal, R. M., Structure prediction and phylogenetic analysis of a functionally diverse family of proteins homologous to the MT-A70 subunit of the human mRNA: m(6) a methyltransferase. J MOL EVOL 55431 (2002).

12 Zheng, G. et al., ALKBH5 is a mammalian RNA demethylase that impacts RNA metabolism and mouse fertility. MOL CELL 4918 (2013).

13 Elsasser, S. J., Noh, K. M., Diaz, N., Allis, C. D. \& Banaszynski, L. A., Histone H3.3 is required for endogenous retroviral element silencing in embryonic stem cells. NATURE $\mathbf{5 2 2}$ 240 (2015).

14 Walsh, C. P., Chaillet, J. R. \& Bestor, T. H., Transcription of IAP endogenous retroviruses is constrained by cytosine methylation. NAT GENET 20116 (1998). 
15 Wang, X. et al., N6-methyladenosine-dependent regulation of messenger RNA stability. NATURE 505117 (2014).

16 Lasman, L. et al., Context-dependent functional compensation between Ythdf m(6)A reader proteins. Genes Dev 341373 (2020).

17 Zaccara, S. \& Jaffrey, S. R., A Unified Model for the Function of YTHDF Proteins in Regulating m(6)A-Modified mRNA. CELL 1811582 (2020).

18 Wang, P., Doxtader, K. A. \& Nam, Y., Structural Basis for Cooperative Function of Mettl3 and Mettl14 Methyltransferases. MOL CELL 63306 (2016).

19 Yue, Y. et al., VIRMA mediates preferential m(6)A mRNA methylation in 3'UTR and near stop codon and associates with alternative polyadenylation. CELL DISCOV 410 (2018).

$20 \mathrm{Li}, \mathrm{X}$. et al., GRID-seq reveals the global RNA-chromatin interactome. NAT BIOTECHNOL 35940 (2017).

21 Dominissini, D. et al., Topology of the human and mouse m6A RNA methylomes revealed by m6A-seq. NATURE 485201 (2012).

22 Meyer, K. D. et al., Comprehensive Analysis of mRNA Methylation Reveals Enrichment in 3 ' UTRs and near Stop Codons. CELL 1491635 (2012).

23 Xiao, Y. et al., An Elongation- and Ligation-Based qPCR Amplification Method for the Radiolabeling-Free Detection of Locus-Specific N(6) -Methyladenosine Modification. Angew Chem Int Ed Engl 5715995 (2018).

$24 \mathrm{Xu}, \mathrm{C}$. et al., Structural basis for selective binding of m6A RNA by the YTHDC1 YTH domain. NAT CHEM BIOL 10927 (2014).

25 Patil, D. P. et al., m(6)A RNA methylation promotes XIST-mediated transcriptional repression. NATURE 537369 (2016).

26 Martienssen, R. \& Moazed, D., RNAi and heterochromatin assembly. Cold Spring Harb Perspect Biol 7 a19323 (2015).

27 Berrens, R. V. et al., An endosiRNA-Based Repression Mechanism Counteracts Transposon Activation during Global DNA Demethylation in Embryonic Stem Cells. CELL STEM CELL 21694 (2017).

28 Zofall, M. et al., RNA Elimination Machinery Targeting Meiotic mRNAs Promotes Facultative Heterochromatin Formation. SCIENCE 33596 (2012).

29 Wang, C. et al., A novel RNA-binding mode of the YTH domain reveals the mechanism for recognition of determinant of selective removal by Mmi1. NUCLEIC ACIDS RES 44969 (2016).

30 Harigaya, Y. et al., Selective elimination of messenger RNA prevents an incidence of untimely meiosis. NATURE 44245 (2006). 


\section{Fig. 1. METTL3 binds endogenous retroviral elements}

341 a: Bar graph showing the overlapping ratios (Jaccard statistics, see Methods) of METTL3 peaks 342 with peaks of different histone modifications.

343 b: Heatmaps showing ChIP-seq enrichments (log2(ChIP/Input)) of METTL3, H3K9me3 and 344 H4K20me3 on METTL3 peaks.

345 c: Heatmap showing co-enrichment of METTL3, H3K9me3 and H4K20me3 on the ERVK 346 subclasses. The 5 most and 5 least METTL3-enriched ERVK subclasses are displayed.

347 d-e: Scatter plots showing the correlation between METTL3 and H3K9me3 (d) or H4K20me3

348 (e) on IAPEz-int $(\mathrm{n}=2,542) . p$ value $=1.7 \mathrm{e}-177(\mathrm{~d})$ and $2.4 \mathrm{e}-151(\mathrm{e})$, two-sided Pearson's 349 correlation test.

350 f: UCSC genome browser snapshots showing the binding pattern of METTL3, histone 351 modifications and input on representative IAPEz elements.

352 Uniquely mapped ChIP-seq reads were used in panel a, b, d, e, f. Uniquely+randomly mapped 353 ChIP-seq reads were used in panel c.

354 Heatmaps were ranked according to METTL3 enrichment in parental cells in descending order 355 in panel $b$. 

a-b: Heatmaps (a) and UCSC genome browser snapshot (b) showing binding patterns of 359 H3K9me3 (Left) and H4K20me3 (Right) on IAPEz-int elements in parental, Mettl3 KO and 360 rescued cell lines with METTL $3^{\mathrm{WT}}$ or METTL3 ${ }^{\mathrm{APPA}}$.

361 c: Boxplot showing RNA levels of the IAPEz-int $(n=2,542)$ in parental, Mettl3 KO and rescued 362 cell lines with METTL3 ${ }^{\mathrm{WT}}$ or METTL3 ${ }^{\mathrm{APPA}}$. $* * * * p<0.0001$ (Exact $p$ values from left to right: 363 4.4e-273, 1.1e-286, 0), two-sided paired t-test.

364 d: RT-qPCR showing relative expression levels of IAPEz-int (Left) and Nxt1 (Right) in parental 365 and Mettl3 KO cell lines treated with Actinomycin D at different time points. Relative RNA 366 levels (normalized to Actin) are normalized to $\mathrm{t}=0$. The mean of three biological replicates \pm 367 s.d. is shown. ${ }^{*} p<0.05,{ }^{*} p<<0.01$, two-sided t-test. Exact $p$ values are provided in the Source 368 Data.

369 Uniquely mapped ChIP-seq and RNA-seq reads were used in panel a, b, c.

370 Heatmaps were ranked according to METTL3 density in parental cells in descending order in 371 panel a.

372 For boxplot in panel c, the middle line, lower and upper hinge of the boxplot correspond to the 373 median, the first and third quartiles, respectively. The whiskers extend from the hinges to no 374 further than $1.5 \times \mathrm{IQR}$ (inter-quartile range) from the hinge. Outlying points that are plotted 375 individually. 
377 Figure 3. METTL3 regulates SETDB1/TRIM28 localization to IAPEz elements

378 a-b: Heatmaps (a), and UCSC genome browser snapshot (b) showing binding patterns of 379 METTL3 on IAPEz-int elements in parental, Mettl3 KO and rescued cell lines with 380 METTL $3^{\text {WT }}$ or METTL3 ${ }^{\text {APPA }}$.

381 c: Western blots showing reciprocal immunoprecipitation of METTL3, SETDB1 and TRIM28.

382 d: Heatmaps showing binding patterns of SETDB1 (Left) and TRIM28 (Right) on IAPEz-int 383 elements in parental and Mettl3 KO cells.

384 e: UCSC genome browser snapshot showing binding patterns of SETDB1 (Upper) TRIM28 385 (Lower) on IAPEz-int elements in parental and Mettl3 KO cells.

386 Uniquely mapped ChIP-seq reads were used in panel a, b, d, e.

387 Heatmaps were ranked according to METTL3 density in parental cells in descending order (a, $388 \mathrm{~d})$.

389 For blots, representative of two independent experiments in panel c. For blots source data, see 390 Supplementary Figure 1. 
Fig. 4. YTHDC1 recruited by METTL3 dependent $\mathbf{m}^{6} \mathrm{~A}$ contributes to METTL3 binding and heterochromatin formation on IAPEz-int

394 a: Aggregation plot showing the average enrichment of $\mathrm{m}^{6} \mathrm{~A}$ and input over IAPEz-int in parental cell line.

396 b: Aggregation plot showing the average enrichment levels of $\mathrm{m}^{6} \mathrm{~A}\left(\log 2\left(\mathrm{~m}^{6} \mathrm{~A} / \mathrm{input}\right)\right)$ over 397 IAPEz-int in parental and Mettl3 KO cell lines.

398 c: Aggregation plot showing the average enrichment of $\mathrm{m}^{6} \mathrm{~A}$ and input over IAPEz-int in Setdb1 399 CKO cell line.

400 d: qPCR showing SELECT results for detecting listed Adenosine positions in IAPEz-int 401 consensus sequence in parental and Mettl3 KO cells. The mean of three biological replicates \pm 402 s.d. is shown. $* p<0.05, * * p<0.01$, two-sided t-test. Exact $p$ values are provided in the Source 403 Data.

404 e: Heatmaps showing YTHDC1 enrichment on the IAPEz-int elements in parental, Mettl3 KO and rescued cell lines with METTL3 ${ }^{\mathrm{WT}}$ or METTL $3^{\mathrm{APPA}}$.

407 in YTHDC1 $1^{\mathrm{WT}}$, Ythdcl $\mathrm{KO}$ and YTHDC1 ${ }^{\mathrm{W} 429 \mathrm{~A}}$ cell lines.

$408 \mathrm{~h}$ : Boxplot showing RNA levels on the IAPEz-int elements $(\mathrm{n}=2,542)$ in YTHDC1 ${ }^{\mathrm{WT}}$, Ythdcl

$409 \mathrm{KO}$ and YTHDC1 ${ }^{\mathrm{W} 429 \mathrm{~A}}$ cell lines. ${ }^{* * * *} p<0.0001$ (Exact $p$ values from left to right: $2.4 \mathrm{e}-78$, 410 7.0e-143), two-sided paired t-test.

411 i: Western blots showing reciprocal immunoprecipitation of METTL3 and YTHDC1.

412 Uniquely mapped reads were used in panel a, b, c, e, f, g, h.

413 Heatmaps were ranked according to METTL3 density in parental cells in descending order in 414 panel e, f, g.

415 For boxplot in panel h, the middle line, lower and upper hinge of the boxplot correspond to the 416 median, the first and third quartiles, respectively. The whiskers extend from the hinges to no 417 further than $1.5 \times$ IQR (inter-quartile range) from the hinge. Outlying points that are plotted 418 individually.

419 For blots, representative of two independent experiments in panel i. For blots source data, see 420 Supplementary Figure 1. 


\section{Antibodies}

424

METTL3 (Bethyl, \#A301-567A, 1/3000 for WB), METTL3 (Abcam, \#ab195352, only used in

425 Extended Data Fig. 1h), METTL3 (Proteintech, \#15073-1-AP, only used in Extended Data Fig.

1h), TRIM28 (Bethyl, \#A300-274A, 1/2000 for WB), SETDB1 (Proteintech, \#11231-1-AP, 1/2000 for WB), H3K9me3 (Active Motif, \#39161), H4K20me3 (Abcam, \#ab9053), H3K4me3 (CST, \#9751), H3K27me3 (CST, \#9733), H3.3 (Millpore, \#09-838), Cas9 (Active motif, \#61757, 1/1000 for WB), m6A (Synaptic Systems, \#202003), YTHDC1 (CST, \#77422, 1/2000 for WB), RBM15 (Proteintech, \#10587-1-AP, 1/2000 for WB), RBM15B (Proteintech, \#22249-1-AP, 1/2000 for WB), METTL14 (Sigma, \#HPA038002, 1/2000 for WB), WTAP (Proteintech, \#10200-1-AP, 1/2000 for WB), ZC3H13 (Bethyl, \#A300-748A, 1/2000 for WB), Virilizer (Bethyl, \#A302-124A, 1/2000 for WB), ALKBH5 (\#ab195377, 1/1000 for WB), HNRNPA2B1 (Proteintech, \#14813-1-AP), HNRNPC (Proteintech, \#11760-1-AP), HNRNPK (Proteintech, \#11426-1-AP).

\section{mES cell culture}

E14Tg2a murine embryonic stem cells (mESCs, gift from Qi-Long Ying, USC) were cultured in Dulbecco's Modified Eagle's Medium (DMEM) supplemented with 10\% fetal FBS (Gibco, \#16000-044), 1\% MEM non-essential amino acid (Gibco, \#11140), 55 mM $\beta$-Mercaptoethanol (Gibco, \#21985-023), 100 U/mL Penicillin/Streptomycin (Hyclone, \#SV30010), 1000 
inhibitor $\mathrm{CH} 99021(3 \mu \mathrm{M})$ at $37^{\circ} \mathrm{C}$ with $5 \% \mathrm{CO}_{2}$.

For EB differentiation, embryoid bodies (EBs) were allowed to form in the absence of LIF by hanging drops containing 1,000 $\mathrm{mES}$ cells/drop on petri dish lids for 2 days, and then collected and transferred to standard mES culture (without LIF and MEK and GSK3 $\beta$ inhibitor) in noncoated petri dishes 5 days.

\section{ChIP-qPCR and ChIP-seq}

451 ChIP assays were performed as described elsewhere ${ }^{31}$. Briefly, chromatin samples were incubated with specific antibodies in the ChIP lysis buffer $(20 \mathrm{mM}$ Tris- $\mathrm{HCl} \mathrm{pH} 8.1,150 \mathrm{mM}$ $\mathrm{NaCl}, 2 \mathrm{mM}$ EDTA, $1 \%$ TritonX-100 and $0.05 \%$ SDS) overnight at $4^{\circ} \mathrm{C}$. The protein-DNA complexes were immobilized on pre-washed protein $\mathrm{A} / \mathrm{G}$ beads. The bound fractions were washed 3 times with the Lysis buffer, and twice with the Low Salt Wash buffer (10 mM Tris$\mathrm{HCl}, 250 \mathrm{mM} \mathrm{LiCl}, 1 \mathrm{mM}$ EDTA, 0.5\% NP-40, 0.5\% Na-deoxylcholate), and once with 10 $\mathrm{mM}$ Tris- $\mathrm{HCl} \mathrm{pH}$ 8.0. Elution and reverse crosslinking were carried out in the Elution buffer

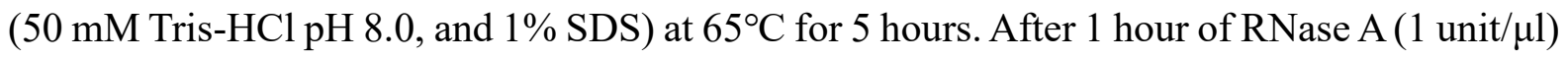
at $37^{\circ} \mathrm{C}$ and Proteinase $\mathrm{K}(1 \mathrm{unit} / \mu \mathrm{l})$ digestion at $55^{\circ} \mathrm{C}$, DNA samples were then purified using PCR extraction kit (QIAGEN, \#28006). The precipitated DNA samples were either analyzed by qPCR (primer sequences are listed in Supplementary Table 1) or prepared for DNA deep sequencing according to manufacturer's guidelines (SWIFT, \#21096). 
Total RNAs from mESCs were isolated using TRIzol reagent (Invitrogen, \#15596018) and treated with TURBO ${ }^{\text {TM }}$ DNase using TURBO DNA-free Kit (Invitrogen, \#AM1970) according to the manufacturer's instruction. cDNAs were synthesized with PrimeScript RT reagent kit (Takara, \#RR037A) containing random primers using $1 \mu \mathrm{g}$ of RNA per sample. RT-qPCR was performed using SYBR Premix ExTaq (Takara, \#RR420Q) with the Roche Lightcycler 480 Instrument II system. Primer sequences are listed in Supplementary Table 1.

Subcellular fractions were collected as following: mESCs were washed once with PBS and lysed in buffer A (10 mM HEPES pH 7.5, $1.5 \mathrm{mM} \mathrm{MgCl}_{2}, 10 \mathrm{mM} \mathrm{KCl,} 0.5 \mathrm{mM}$ DTT, $1 \mathrm{mM}$ PMSF and 1x Protease Inhibitor Cocktail) on ice for $15 \mathrm{~min}$, then NP-40 was added to a final concentration of $0.25 \%$ for another $5 \mathrm{~min}$. Nuclei were collected by centrifugation $(2,000 \mathrm{rpm}$, $3 \mathrm{~min}, 4^{\circ} \mathrm{C}$ ) with the supernatant as cytoplasmic fraction. The nuclei were re-suspended in buffer C (20 mM HEPES, pH 7.5, 10\% Glycerol, $0.42 \mathrm{M} \mathrm{KCl,} 4 \mathrm{mM} \mathrm{MgCl} 2,0.2 \mathrm{mM}$ EDTA, $0.5 \mathrm{mM}$ DTT, $1 \mathrm{mM}$ PMSF and 1x Protease Inhibitor Cocktail). After 30 min incubation on ice, nuclear extract was collected by high speed centrifugation $\left(13,000 \mathrm{rpm}, 15 \mathrm{~min}, 4^{\circ} \mathrm{C}\right)$ as nuclear fraction. Insoluble chromatin fraction was re-suspended with buffer A. RNAs in different subcellular fractions were isolated using TRIzol reagent (Invitrogen, \#15596018) and treated with TURBO ${ }^{\text {TM }}$ DNase using TURBO DNA-free Kit (Invitrogen, \#AM1970) according to the manufacturer's instruction. 


\section{Construction of stable cell lines and knockdown}

486

487

488

489

490

491

492

493

494

495

496

497

498

499

500

501

502

503

504

505

CRISPR-Cas9 gene targeting was carried out as previously described ${ }^{32}$ and the single knockout clones were isolated and then confirmed by Western blot showing undetectable protein. Mettl3, Mettl14 and Alkbh5 KO cells were created by introduction of one sgRNA. Rbm15/15b double KO cells were created by simultaneous introduction of two sgRNAs targeting $R b m 15$, and two sgRNAs targeting Rbm15b. Guiding RNA sequences and donor sequence used are listed in Supplementary Table 2 .

Setdb1 CKO mESCs were kindly provided by Yoichi Shinkai (REKIN). Setdb1 CKO mESCs carried one null and one floxed allele of Setdb1, which could be deleted upon CRE-mediated excision. Treatment with 4-hydroxytamoxifen (4-OHT) induced the activation of ligand binding domain of estrogen receptor and CRE recombinase fusion protein, leading to conditional deletion of the remaining allele ${ }^{8}$. For $4-\mathrm{OHT}$ treatment, mESCs were cultured in ES medium with $800 \mathrm{nM}$ 4-OHT for 4 days, and further cultured without 4-OHT for 2 days. Depletion of SETDB1 was validated by Western blotting.

For rescued cells, cDNA of full-length murine METTL3 WT (NCBI RefSeq: NM_019721.2) or METTL3 mutation (DPPW motif to APPA, W475A, N477A) were cloned into the pPBCAG-IRES-Pac plasmid (Puromycine resistant). These plasmids were individually cotransfected into mESCs with pCMV-PBase plasmid in a 1:1 ratio using Lipofectamine 2000 (Invitrogen, \#11668-019) according to the manufacturer's instruction. Medium was replaced 

selection for 5 days, the surviving mESCs were pooled as stable rescued cell lines.

509 Construction of YTHDC1 ${ }^{\mathrm{WT}}$, Ythdc1 KO, and YTHDC1 ${ }^{\mathrm{W} 429 \mathrm{~A}}$ mESCs in auxin-inducible 510 degron (AID) system is shown in Extended Data Fig. 7f. Briefly, AID-tag-fused YTHDC1 511 (NCBI Reference Sequence: NM_177680.4, resistant to gRNA, c.1264_1290CATGGCGGATCTCCTATACAC>CACGGAGGCAGCCCCATCCAT) was cloned into the pPB-CAG-IRES-Pac plasmid (Puromycine resistant), and TIR1 was cloned into the pPB-CAG-IRES-Pac plasmid (Blasticidin S resistant). mESCs were randomly integrating the expression cassettes expressing TIR1 and AID-tag-fused YTHDC1 into the genome, followed by knocking out the endogenous Ythdcl with gRNA, or knocking in the YTHDC $1^{\text {W429A }}$ mutation into the endogenous $Y t h d c 1$ with gRNA and donor. Single colonies were picked and positive colonies were identified by PCR genotyping.

For the dCas9 experiment in Extended Data Fig. 41-n and 8m-n, the gRNA expression plasmids were cloned by inserting annealed oligos into the modified pgRNA plasmid (Addgene plasmid: 44248). The PiaggyBac-dCas9-METTL3 ${ }^{\mathrm{WT}} / \mathrm{METTL}^{\mathrm{APPA}}$ or ${ }^{\mathrm{YTHDC} 1}$ were cloned by replacing the DNMT3A fragment (138-dCas9-DNMT3A, addgene plasmid: 84570) ${ }^{33}$. Lentiviruses expressing gRNAs were produced by co-transfection of gRNA plasmids (Ctrl. or 
transfection was collected. Mettl3 KO mESCs were seeded in a 6-well plate and infected with

each gRNA lentivirus supernatant in the presence of $5 \mu \mathrm{g} / \mathrm{mL}$ polybrene. Medium was replaced by fresh media with Puromycine $(2 \mu \mathrm{g} / \mathrm{ml})$ for 5 days, the surviving mESCs were pooled as stably infected mESCs. To generate stable cell lines with integrated Doxycycline-inducible transgenes for dCas9-METTL3 or dCas9-YTHDC1, the dCas9-METTL3 or dCas9-YTHDC1 plasmids were co-transfected into mESCs expressing gRNA with pCMV-PBase plasmid and selected with G418 (400 $\mu \mathrm{g} / \mathrm{ml})$ for 10 days. Doxycycline was added to the cell culture media to a final concentration of $2 \mu \mathrm{g} / \mathrm{mL}$ for 5 days for ChIP experiments.

For the dCas9 experiment in Extended Data Fig. 8o-p, the Mettl3 KO+METTL3 ${ }^{\text {APPA }}$ stable cell line (Blasticidin S resistant) was first generated. METTL3 ${ }^{\text {APPA }}$ mutation was cloned into the pPB-CAG-IRES-Pac plasmid (Blasticidin S resistant). This plasmid was co-transfected into mESCs with pCMV-PBase plasmid in a 1:1 ratio using Lipofectamine 2000 (Invitrogen, \#11668-019) according to the manufacturer's instruction. Medium was replaced by fresh media with $10 \mu \mathrm{g} / \mathrm{mL}$ Blasticidin S 48 hours post-transfection. After continuous selection for 7 days, the surviving mESCs were pooled as stable rescued cell lines. Mettl3 KO+METTL3 ${ }^{\text {APPA }}$ mESCs were seeded in a 6-well plate and infected with gRNA lentivirus in the presence of 5 $\mu \mathrm{g} / \mathrm{mL}$ polybrene. Medium was replaced by fresh media with Puromycine $(2 \mu \mathrm{g} / \mathrm{ml})$ for 5 days, the surviving mESCs were pooled as stably infected mESCs. To generate stable cell lines with integrated Doxycycline-inducible transgenes for dCas9-YTHDC1, YTHDC1 plasmid was cotransfected into mESCs expressing gRNA with pCMV-PBase plasmid and selected with G418 (400 $\mu \mathrm{g} / \mathrm{ml}$ ) for 10 days. Doxycycline was added to the cell culture media to a final 
concentration of $2 \mu \mathrm{g} / \mathrm{mL}$ for 5 days for ChIP experiments.

SiRNA-mediated gene knockdown was performed using Lipofectamine RNAiMAX

552

553

554

555

556

557

558

559

560

561

562

563

564

565

566

567 (Invitrogen, \#13778-150) with siRNA at $25 \mathrm{nM}$ final concentration. Cells were harvested after 3 days. SiRNA oligonucleotides are listed at Supplementary Table 3.

\section{Isolation of mRNA for QQQ and HPLC analysis}

mRNA was isolated from $1 \mu \mathrm{g}$ total RNA with Oligo d(T) 25 magnetic beads (NEB, \#S1419S), followed with RiboMinus Kit (Invitrogen, \#A15026). Purified mRNA was digested by nuclease P1 (Thermo, \#18009027) in $25 \mu \mathrm{l}$ of buffer containing $25 \mathrm{mM} \mathrm{NaCl}$ and $2.5 \mathrm{mM}$ of $\mathrm{ZnCl}_{2}$ at $42^{\circ} \mathrm{C}$ for 2 hours, which was followed by addition of $\mathrm{NH}_{4} \mathrm{HCO}_{3}(1 \mathrm{M}, 3 \mu \mathrm{l})$ and alkaline phosphatase (Sigma, $\# \mathrm{P} 4252$ ) and additional incubation at $37^{\circ} \mathrm{C}$ for 2 hous. Samples were then diluted to $60 \mu 1$ and $5 \mu 1$ of solution was loaded into liquid chromatography-tandem mass spectrometry (LC-MS/MS) (Agilent 6410 QQQ Triple-Quadrupole Mass Spectrometer).

\section{The SELECT detection assay}

SELECT (single base elongation- and ligation-based qPCR amplification method) was carried out as previously described ${ }^{23}$. Briefly, $1 \mu \mathrm{g}$ total RNA was mixed with $40 \mathrm{nM}$ Up Primer, 40 nM Down Primer and $5 \mu \mathrm{M}$ dNTP in $17 \mu 1$ 1×CutSmart buffer (NEB, \#B7204S). The RNA and primers were annealed by incubating mixture at a temperature gradient: $90^{\circ} \mathrm{C}$ for $1 \mathrm{~min}, 80^{\circ} \mathrm{C}$ 
570 Subsequently, a $3 \mu \mathrm{l}$ of mixture containing 0.01 U Bst 2.0 DNA polymerase, 0.5 U SplintR

571 ligase and $10 \mathrm{nmol}$ ATP was added in the former mixture to the final volume $20 \mu \mathrm{l}$. The final

572 reaction mixture was incubated at $40^{\circ} \mathrm{C}$ for $20 \mathrm{~min}$, denatured at $80^{\circ} \mathrm{C}$ for $20 \mathrm{~min}$. The qPCR

573 reaction was performed using SYBR Premix Ex Taq (Takara, \#RR420Q) with the Roche

574 Lightcycler 480 Instrument II system. IAPEz-int consensus sequence was downloaded from

575 Repbase. Primer sequences are listed in Supplementary Table 4.

\section{$577 \quad$ In vitro $\mathbf{m}^{\mathbf{6}} \mathbf{A}$ methylation assay}

578 The in vitro $\mathrm{m}^{6} \mathrm{~A}$ methylation assay was carried out in a $20 \mu \mathrm{l}$ reaction mixture containing $0.5 \mu \mathrm{g}$ METTL3/METTL14 protein (Active motif, \#31970), 0.5 $\mu \mathrm{g}$ RNA oligonucleotides, $20 \mathrm{mM}$ Tris (pH 7.5), 0.01\% Triton-X, $1 \mathrm{mM}$ DTT, $50 \mathrm{mM} \mathrm{ZnCl}_{2}, 0.2 \mathrm{U} / \mathrm{mL}$ RNasin, $1 \%$ glycerol, and 460 $\mathrm{nM}\left[{ }^{3} \mathrm{H}\right]-\mathrm{SAM}$. Each in vitro $\mathrm{m}^{6} \mathrm{~A}$ methylation reaction was incubated at room temperature for 1 hour. RNA was extract with Trizol and dissolved in $20 \mu 1 \mathrm{H}_{2} \mathrm{O}$. The counts per minute (C.P.M.) of the RNA was measured in a scintillation counter.

\section{WGBS library preparation}

The bisulfate conversion was performed using the EZ DNA Methylation Gold kit from Zymo 
Specific total RNA library preparation was performed using NEBNext Ultra Directional RNA

591 Library Prep Kit for Illumina (NEB, \#E7420S).

592

593

\section{ChIRP-seq}

594 ChIRP was performed according to the previous work with some modifications ${ }^{34}$. mESCs were

595

596

597

598

599

600

601

602

603

604

605

606

607

608

609 crosslinked with $3 \%$ formaldehyde for $30 \mathrm{~min}$ at room temperature. Crosslinking was then quenched with $0.125 \mathrm{M}$ glycine for $5 \mathrm{~min}$. Chromatin was then solubilized by sonicating in lysis buffer (50 mM Tris 7.0, $10 \mathrm{mM}$ EDTA, 1\% SDS, $0.5 \mathrm{mM}$ DTT, and RNase Inhibitors). Chromatin is diluted in two times volume of hybridization buffer ( $750 \mathrm{mM} \mathrm{NaCl}, 1 \% \mathrm{SDS}, 50$ $\mathrm{mM}$ Tris 7.0, $1 \mathrm{mM}$ EDTA, 15\% Formamide, $0.5 \mathrm{mM}$ DTT, and RNase Inhibitors). 10 pmol probes (20 probes mixed equally, sequences are listed in Supplementary Table 5) were added to diluted chromatin, which was mixed by end-to-end rotation at $37^{\circ} \mathrm{C}$ for 6 hours, followed by the addition of Streptavidin beads for another $45 \mathrm{~min}$ at $37^{\circ} \mathrm{C}$. The bound fractions were washed 5 times with wash buffer (2x SSC, 0.5\% SDS, add DTT and RNase Inhibitors). Elution and reverse crosslinking were carried out at $65^{\circ} \mathrm{C}$ overnight. After 1 hour of RNase A (1unit/ $\left.\mu \mathrm{l}\right)$ at $37^{\circ} \mathrm{C}$ and Proteinase $\mathrm{K}(1 \mathrm{unit} / \mu \mathrm{l})$ digestion at $55^{\circ} \mathrm{C}$, DNA samples were then purified using PCR extraction kit (QIAGEN, \#28006). The precipitated DNA samples were prepared for DNA deep sequencing according to manufacturer's guidelines (SWIFT, \#21096). 
611 For transcription inhibition ${ }^{35}$, mESCs were treated with $1 \mu \mathrm{M}$ Flavopiridol, or $1 \mu \mathrm{M}$ Triptolide 612 added directly to the culture media and cells were incubated with the drug for 12 hours at $37^{\circ} \mathrm{C}$, 613 or $10 \mu \mathrm{M} \alpha$-Amanitin for 24 hours at $37^{\circ} \mathrm{C}$ for $\mathrm{ChIP}$ experiments. mESCs were treated with 2.5 $614 \mu \mathrm{g} / \mathrm{ml}$ Actinomycin D directly to the culture media and cells were incubated with the drug for different time points $(1,3,5,7,10$ hours $)$ at $37^{\circ} \mathrm{C}$ for the RNA decay experiments.

\section{Co-Immunoprecipitation}

mESCs were washed once with PBS and lysed in buffer A (10 mM HEPES pH 7.5, $1.5 \mathrm{mM}$ $\mathrm{MgCl}_{2}, 10 \mathrm{mM} \mathrm{KCl}, 0.5 \mathrm{mM}$ DTT, $10 \mathrm{mM}$ Nethylmaleimide (NEM), 1mM PMSF and 1x Protease Inhibitor Cocktail) on ice for $15 \mathrm{~min}$, then NP-40 was added to a final concentration of $0.25 \%$ for another $5 \mathrm{~min}$. Nuclei were collected by centrifugation $\left(2,000 \mathrm{rpm}, 3 \mathrm{~min}, 4^{\circ} \mathrm{C}\right)$ and re-suspended in buffer C (20 mM HEPES, pH 7.5, 10\% Glycerol, $0.42 \mathrm{M} \mathrm{KCl,} 4 \mathrm{mM}$ $\mathrm{MgCl}_{2}, 0.2 \mathrm{mM}$ EDTA, $0.5 \mathrm{mM}$ DTT, $10 \mathrm{mM}$ Nethylmaleimide (NEM), $1 \mathrm{mM}$ PMSF and Protease Inhibitor Cocktail). After 30 min incubation on ice, nuclear extract was collected by high speed centrifugation $\left(13,000 \mathrm{rpm}, 15 \mathrm{~min}, 4^{\circ} \mathrm{C}\right)$ as nuclear extract $\mathrm{A}$. Insoluble chromatin

626 fraction was re-suspended with buffer A ( 2 X volumes of buffer C) with DNase I. After 30 min 627 incubation at $37^{\circ} \mathrm{C}$, the soluble fraction was collected by high speed centrifugation $(13,000 \mathrm{rpm}$, $\left.15 \mathrm{~min}, 4^{\circ} \mathrm{C}\right)$ as nuclear extract $\mathrm{B}$. Nuclear extract $\mathrm{A}$ and nuclear extract $\mathrm{B}$ were mixed and incubated with indicated antibody or IgG for 6 hours at $4^{\circ} \mathrm{C}$, followed by the addition of 
631

632

633

634

635

636

637

638

639

640

641

642

643

644

645

646

647

648

649

650

651

buffer (Mixture of buffer A and buffer $\mathrm{C}$ in a ratio of 2:1). SDS buffer was directly added to the beads and boiled for $10 \mathrm{~min}$. The samples were loaded on SDS-PAGE gels and subjected to immunoblotting using indicated antibodies.

\section{MeRIP-seq}

For chromatin RNA MeRIP-seq, chromatin RNA was collected as described above. $20 \mu \mathrm{g}$ RNA was sonicated to 100-200nt fragments by Bioruptor Plus sonicator device (Diagenode) and incubated with $5 \mu \mathrm{g}$ anti-m ${ }^{6} \mathrm{~A}$ antibody in $1 \mathrm{x}$ IP buffer $(10 \mathrm{mM}$ Tris-HCl, pH 7.4, $150 \mathrm{mM}$ $\mathrm{NaCl}, 0.1 \% \mathrm{NP}-40$ ) for 2 hours at $4^{\circ} \mathrm{C}$. The $\mathrm{m}^{6} \mathrm{~A}-\mathrm{IP}$ mixture was then incubated with Dynabeads protein $\mathrm{A} / \mathrm{G}$ for an additional 2 hours at $4^{\circ} \mathrm{C}$ on a rotating wheel. After washing 3 times with 1 $\mathrm{x}$ IP buffer, the bound RNA was isolated with TRIzol reagent. The purified RNA fragments from MeRIP and Input RNA were first treated with Ribo-off rRNA Depletion Kit (Vazyme, \#N406), followed with library construction using NEBNext Ultra Directional RNA Library Prep Kit for Illumina (NEB, \#E7420) according to manufacturer's guidelines.

\section{ChIP-seq analysis}

Raw reads were trimmed using Trim_galore (v0.6.4_dev) and aligned to the mm10 genome using Bowtie2 (v2.2.5) ${ }^{36}$ to report best alignment with MAPQ. Unique reads were filtered by MAPQ $>20$. PCR duplicates were removed using samtools (v1.7) ${ }^{37}$ rmdup. Genome coverage bedGraph files for UCSC genome browser were generated by deeptools (v3.0.2) ${ }^{38}$ bamCoverage with the parameters “-of bedgraph --normalizeUsing RPKM --binSize 5”. Peaks 
652

653

654

655

656

657

658

659

660

661

662

663

664

665

666

667

668

669

670

671

672

were generated by macs2 $(2.1 .4)^{39}$ callpeak with parameters “-p 0.00001 --nomodel”. Peak annotation was carried out using Homer $(\mathrm{v} 4.8 .2)^{40}$ annotatePeaks.pl. The Jaccard statistic representing the ratio of the intersection of two sets to the union of the two sets is calculated using bedtools (v2.29.2) ${ }^{41}$. The relative distances between each interval in one set and the two closest intervals in another set is calculated using bedtools (v2.29.2) ${ }^{41}$. Genome coverage bigwig files for heatmap and aggregation plot were generated by deeptools (v3.0.2) ${ }^{38}$ bamCoverage with the parameter “--normalizeUsing RPKM --binSize 5". Heatmaps were generated by deeptools (v3.0.2) $)^{38}$ computeMatrix and plotHeatmap. Aggregation plots were generated by deeptools $(\mathrm{v} 3.0 .2)^{38}$ computeMatrix and plotProfile. ChIP-seq density on repetitive elements were calculated by Homer $(\mathrm{v} 4.8 .2)^{40}$ analyzeRepeats.pl. Boxplot were generated by R boxplot. METTL3 peak is listed in Supplementary Table 6.

\section{WGBS analysis}

Raw reads were trimmed 10 bps off at the 5' end and 3' end and then mapped to mm10 genome using bsmap (v2.90) ${ }^{42}$ with the parameters “-p 10 -w 50 -v 0.1 ”. Methylation ratios were calculated by methratio.py with the parameters "-t 2 -m 5 -z". Methylation over a given genomic feature was calculated by averaging the individual methylation levels of CpGs and only features with at least 10 covered CpGs were used. Boxplot were generated by R boxplot.

\section{Total RNA-seq analysis}

Raw reads were trimmed using Trim_galore (v0.6.4_dev) and aligned to the mm10 genome 
673

674

675

676

677

678

679

680

681

682

683

684

685

686

687

688

689

690

691

692

693

using TopHat (v2.1.1) ${ }^{43}$ with parameter “--max-multihits 1". PCR duplicates were removed using samtools (v1.7) ${ }^{37}$ rmdup. Strand specific reads were separated by samtools $(\mathrm{v} 1.7)^{37}$ view with specific flags $99,147,83,163$. Strand specific density on repetitive elements were calculated by Homer (v4.8.2) ${ }^{40}$ analyzeRepeats.pl with the parameter "strand". Boxplot were generated by R boxplot.

\section{ChIRP-seq analysis}

Raw reads were trimmed using Trim_galore (v0.6.4_dev) and aligned to the mm10 genome using Bowtie2 (v2.2.5) ${ }^{36}$ to report best alignment with MAPQ. PCR duplicates were removed using samtools (v1.7) ${ }^{37}$ rmdup. Genome coverage bigwig files aggregation plot were generated by deeptools (v3.0.2) $)^{38}$ bamCoverage with the parameter “--normalizeUsing RPKM --binSize 5". Aggregation plots were generated by deeptools (v3.0.2) $)^{38}$ computeMatrix and plotProfile.

\section{MeRIP-seq analysis}

Raw reads were trimmed using Trim_galore (v0.6.4_dev) and aligned to the mm10 genome using using TopHat (v2.1.1) with parameter “--max-multihits 1". PCR duplicates were removed using samtools ${ }^{37}$ (v1.7) rmdup. Strand specific reads were separated by samtools ${ }^{37}$ (v1.7) view with specific flags 99, 147, 83, 163. Genome coverage bedGraph files for UCSC genome browser were generated by deeptools (v3.0.2) 38 bamCoverage with the parameters “of bedgraph --normalizeUsing RPKM --binSize 5". $\mathrm{m}^{6} \mathrm{~A} / \mathrm{Input}$ ratio bigwig files for aggregation plot were generated by deeptools (v3.0.2) ${ }^{38}$ bamCompare with the parameter "-- 
694

695

696

697

698

699

700

701

702

703

704

705

706

707

708

709

710

711

712

713

714

715

716

717

718

719

720

721

722

723

724

725

726

727

normalizeUsing RPKM --binSize 5". Aggregation plots were generated by deeptools (v3.0.2) ${ }^{38}$ computeMatrix and plotProfile. $\mathrm{m}^{6} \mathrm{~A}$ peak calling was performed using a "sliding window" method slightly modified from the previous study ${ }^{22}$. Briefly, reads numbers of IP and Input were calculated on every $25 \mathrm{bp}$ window across genome. Windows with normalized IP/Input density $\geq 2$, Fisher's Exact Test $\mathrm{p}$-value $<0.05$ were selected. Adjacent windows were merged using bedtools (v2.29.2) ${ }^{41}$ and the merged regions with size $\geq 100 \mathrm{bp}$ were determined as $\mathrm{m}^{6} \mathrm{~A}$ peak. Consensus motifs are called using Homer (v4.8.2 $)^{40}$ findMotifsGenome.pl with the parameter "-rna".

31 Lan, F. et al., A histone $\mathrm{H} 3$ lysine 27 demethylase regulates animal posterior development. NATURE 449689 (2007).

32 Maeder, M. L. et al., CRISPR RNA-guided activation of endogenous human genes. NAT METHODS 10977 (2013).

33 Liu, X. S. et al., Editing DNA Methylation in the Mammalian Genome. CELL 167233 (2016).

34 Chu, C., Qu, K., Zhong, F. L., Artandi, S. E. \& Chang, H. Y., Genomic maps of long noncoding RNA occupancy reveal principles of RNA-chromatin interactions. MOL CELL $\mathbf{4 4}$ 667 (2011).

35 Bensaude, O., Inhibiting eukaryotic transcription: Which compound to choose? How to evaluate its activity? Transcription 2103 (2011).

36 Langmead, B. \& Salzberg, S. L., Fast gapped-read alignment with Bowtie 2. NAT METHODS 9357 (2012).

$37 \mathrm{Li}$, H. et al., The Sequence Alignment/Map format and SAMtools. BIOINFORMATICS 25 2078 (2009).

38 Ramirez, F. et al., deepTools2: a next generation web server for deep-sequencing data analysis. NUCLEIC ACIDS RES $44 \mathrm{~W} 160$ (2016).

39 Zhang, Y. et al., Model-based analysis of ChIP-Seq (MACS). GENOME BIOL 9 R137 (2008).

40 Heinz, S. et al., Simple combinations of lineage-determining transcription factors prime cis-regulatory elements required for macrophage and B cell identities. MOL CELL 38576 (2010).

${ }^{41}$ Quinlan, A. R. \& Hall, I. M., BEDTools: a flexible suite of utilities for comparing genomic features. BIOINFORMATICS 26841 (2010).

${ }^{42} \mathrm{Xi}, \mathrm{Y} . \& \mathrm{Li}, \mathrm{W} ., \mathrm{BSMAP}$ : whole genome bisulfite sequence MAPping program. BMC 
72943 Trapnell, C., Pachter, L. \& Salzberg, S. L., TopHat: discovering splice junctions with 730 RNA-Seq. BIOINFORMATICS 251105 (2009). 


\section{Acknowledgements}

734 We thank Danesh Moazed (Harvard Medical School) and Erdem Sendinc (Boston Children's

735 Hospital) for critical reading of the manuscript and suggestions. We thank Jiekai Chen

736 (Guangzhou Institutes of Biomedicine and Health, Chinese Academy of Sciences) for 737 suggestions and for sharing unpublished results. We thank Yoichi Shinkai (REKIN) for sharing 738 the Setdb1 CKO cell line. H.S. was supported by Shanghai Rising-Star Program 739 (19QA1401300) and National Science Foundation of China (81874157, 32070649, 31601060).

740 W.X. was supported by National Science Foundation of China (31900469). Y.S. is an American 741 Cancer Society Research Professor.

\section{Author contributions}

W.X. and H.S. carried out most of the experiments and bioinformatics analyses described in this manuscript. J.L. carried out YTHDC1 ${ }^{\mathrm{W} 429 \mathrm{~A}}$ identification and genotyping. C.H. carried out LC-MS/MS analysis of $\mathrm{m}^{6} \mathrm{~A}$. J.W. and J.D. provided discussions and advice on coimmunoprecipitation. L.T. provided discussions and advice on mES cell culture. L.W., J.W. and B.R. provided discussions and advice on ChIP. H.M. and F.W. provided discussion and advice on $\mathrm{m}^{6} \mathrm{~A}$ RIP and bioinformatics analyses, respectively. W.X., H.S. and Y.S. conceived the project and co-wrote the manuscript. H.S and Y.S. directed all the experiments with input from Y.G.S. 
755 Y.S. is a co-founder and holds equity of Constellation Pharmaceuticals, Inc. and Athelas

756 Therapeutics, Inc. Y.S. also holds equity of Imago Biosciences and is a consultant for Active 757 Motif, Inc.

758

759 Correspondence and requests for materials should be addressed to Y.S.

760 (yang.shi@ludwig.ox.ac.uk) or H.S. (hongjieshen@fudan.edu.cn).

761

\section{Data availability}

763 The next-generation-sequencing data generated by this study have been deposited to GEO

764 database under accession number GEO: GSE126243. 
767 a: Accumulative plots showing the relative distances between peaks of METTL3 and histone 768 modifications.

769 b: Bar graph showing the overlapping ratios (calculated as Jaccard statistics, see Methods) of 770 METTL3 peaks with repetitive elements.

771 c: Accumulative plots showing the relative distances between METTL3 peaks and repetitive 772 elements.

773 d: Heatmaps showing enrichment of METTL3, H3K9me3 and H4K20me3 on repetitive 774 elements.

775 e: Scatter plots showing the correlation between the enrichment levels of METTL3 and 776 H3K9me3 (Left) or H4K20me3 (Right) on different subtypes ( $n=277)$ of ERVK 777 retrotransposons. Two-sided Pearson's correlation test.

778 f: Schematic representation of stitching adjacent IAPEz fragments.

779 g: Aggregation plots showing METTL3, H3K9me3, H4K20me3 and Input patterns on IAPEz780 int.

781 h: Validation of METTL3 binding on IAPEz using three independent METTL3 antibodies. The 782 mean of three biological replicates \pm s.d. is shown.

783 i: Schematic representation of uniquely mapped reads and randomly mapped un-unique reads.

$784 \mathrm{j}$ : Aggregation plots and heatmaps showing enrichment of METTL3, H3K9me3 and 785 H4K20me3 on the IAPEz-int elements with only uniquely mapped reads or 786 uniquely+randomly mapped reads.

787 Uniquely mapped ChIP-seq reads were used in panel a, b, c, e. Uniquely and randomly mapped 788 ChIP-seq reads were used in panel $\mathrm{d}$, g.

789 Heatmaps were ranked according to METTL3 enrichment in parental cells in descending order 790 (j).

791 MTA and MaSat used in ChIP-qPCR are examples of repetitive elements unbound by METTL3. 792 


\section{Extended Data Fig. 2. METTL3 is required for heterochromatin formation} a: Western blots showing METTL3 protein levels in parental, Mettl3 KO and rescued cell lines with METTL3 ${ }^{\text {WT }}$ or METTL3 ${ }^{\text {APPA }}$. b: Bar plots showing $\mathrm{m}^{6} \mathrm{~A} / \mathrm{A}$ ratio on polyA RNAs in parental, Mettl3 $\mathrm{KO}$ and rescued cell lines with METTL3 $^{\text {WT }}$ or METTL3 ${ }^{\text {APPA }}$. The mean of three biological replicates \pm s.d. is shown. c: ChIP-qPCR showing binding patterns of H3K9me3 (Left) and H4K20me3 (Right) on IAPEz-int elements in parental, Mettl3 KO and rescued cell lines with METTL $3{ }^{\text {WT }}$ or METTL3 ${ }^{\text {APPA }}$. The mean of three biological replicates \pm s.d. is shown in ChIP-qPCR. ${ }^{*} p<0.05$, ${ }^{* *} p<0.01$. two-sided t-test. Exact $p$ values are provided in the Source Data. int elements $(\mathrm{n}=2,542)$ in parental, Mettl3 $\mathrm{KO}$ and rescued cell lines with wildtype or catalytically mutated METTL3. $* * * * p<0.0001$ (Exact $p$ values from left to right: $0,0,0,1.1 \mathrm{e}-$ $118,6.2 \mathrm{e}-269,0)$, two-sided paired t-test.

806 e: Aggregation plots showing enrichment levels of H3K9me3 (Left) and H4K20me3 (Right) on IAPEz-int elements in parental, Mettl3 $\mathrm{KO}$ and rescued cell line. f-h: Heatmaps (f), boxplots (g) and aggregation plots (h) showing enrichment levels of H3K9me3 on IAPEz-int elements $(\mathrm{n}=2,542)$ in parental and Alkbh5 KO cell lines. **** $p<0.0001$, two-sided paired t-test.

811 i: Western blots showing ALKBH5 protein levels in parental and Alkbh5 KO cell lines. j-k: Boxplot showing density fold changes (log2(Mettl3 KO/Parental)) of H3K9me3 (i) and H4K20me3 (j) on different types of repetitive elements upon Mettl3 KO. $p=0.00014$, two814 sided paired t-test.

815 Uniquely mapped ChIP-seq reads were used in panel d, f, g, j, k. Uniquely+randomly mapped 816 ChIP-seq reads were used in panel e, $h$.

817 Heatmaps were ranked according to METTL3 density in parental cells in descending order (f). For the boxplots in panel $\mathrm{d}, \mathrm{g}, \mathrm{j}, \mathrm{k}$, the middle line, lower and upper hinge of the boxplot correspond to the median, the first and third quartiles, respectively. The whiskers extend from the hinges to no further than $1.5 \times \mathrm{IQR}$ (inter-quartile range) from the hinge. Outlying points that are plotted individually.

822 For blots, representative of two independent experiments in panel a, i. For blots source data, see Supplementary Figure 1. 


\section{Extended Data Fig. 3. METTL3 is required for heterochromatin formation}

827 a: Boxplots showing enrichment levels of H3.3 on IAPEz-int elements $(n=2,542)$ in parental, 828 Mettl3 KO and rescued cell lines with METTL3 ${ }^{\mathrm{WT}}$ or METTL3 ${ }^{\mathrm{APPA}}$. $* * * *^{\mathrm{K}}<<0.0001$ (exact $p$ 829 values from left to right: 4.2e-218, 2.7e-274, 3.9e-294), two-sided paired t-test.

830 b: Boxplots showing CpG methylation ratios on IAPEz-int elements $(n=2,542)$ in parental, 831 Mettl3 KO and rescued cell lines with with METTL3 ${ }^{\mathrm{WT}}$ or METTL3 ${ }^{\mathrm{APPA}}$. $* * * * p<0.0001$ (exact $832 p$ values from left to right: 4.87e-111, 1.5e-150, 3.9e-294,0), two-sided paired t-test.

833 c: Boxplot showing density fold changes ( $\log 2($ Mettl3 KO/Parental)) of H3.3 on different types 834 of repetitive elements upon Mettl3 KO.

$835 \mathrm{~d}$ : Boxplot showing CpG methylation changes on different types of repetitive elements upon 836 Mettl3 KO. Only elements with at least 10 covered CpGs were used. e: RT-qPCR showing RNA levels of IAPEz-int in parental, Mettl3 KO and rescued cell lines with METTL3 ${ }^{\mathrm{WT}}$ or METTL3 ${ }^{\mathrm{APPA}}$. The mean of three replicates \pm s.d. is shown. $* p<0.05$, ** $p<0.01$, two-sided t-test. Exact $p$ values are provided in the Source Data.

840 f: Boxplot showing density fold changes $(\log 2($ Mettl3 KO/Parental)) of RNAs of different 841 types of repetitive elements upon Mettl3 KO.

842 g-j: Scatter plots showing correlation between METTL3 (g), H3K9me3 (h), H4K20me3 (i), 843 DNA methylation ( $\mathrm{j})$ and RNA expression level on IAPEz-int $(\mathrm{n}=2,542)$. Two-sided Pearson's 844 correlation test.

845 k: Boxplot showing RNA levels of the IAPEz-int $(\mathrm{n}=2,542)$ in parental, Mettl3 KO and $846 Y$ thdf1/2/3 KO cell lines revealed by PolyA RNA-seq (GSE147849). **** $p<0.0001$ (exact $p$ 847 values from left to right: 1.1e-52, 7.3e-37), two-sided paired t-test.

848 Uniquely mapped ChIP-seq reads were used in panel a, b, c, d, f, g, h, i, j, k.

849 For the boxplots in panels a, b, c, d, f and k, the middle line, lower and upper hinge of the 850 boxplot correspond to the median, the first and third quartiles, respectively. The whiskers 851 extend from the hinges to no further than $1.5 \times \mathrm{IQR}$ (inter-quartile range) from the hinge. 852 Outlying points that are plotted individually. 
Extended Data Fig. 4. METTL3 chromatin binding is dependent on its own catalytic activity

856 a: ChIP-qPCR showing binding patterns of METTL3 on IAPEz-int elements in parental, Mettl3 $857 \mathrm{KO}$ and rescued cell lines with METTL3 ${ }^{\mathrm{WT}}$ or METTL3 ${ }^{\mathrm{APPA}}$. The mean of three biological 858 replicates \pm s.d. is shown. $* p<0.05, * * p<0.01$, two-sided t-test. Exact $p$ values are provided 859 in the Source Data.

860 b: Aggregation plots showing METTL3 enrichment levels on IAPEz-int in parental, Mettl3 KO 861 and rescued cell lines with METTL3 ${ }^{\mathrm{WT}}$ or METTL3 ${ }^{\mathrm{APPA}}$.

862 c: Western blot showing interactions of METTL14 with reintroduced METTL3 (METTL3 ${ }^{\text {WT }}$ 863 or METTL3 ${ }^{\mathrm{APPA}}$ ) in Mettl3 KO cells.

864 d: Aggregation plots showing METTL3 enrichment levels on IAPEz-int in Mettl3 KO rescued 865 cells with METTL3 $^{\text {WT }}$, METTL3 ${ }^{\text {W475A }}$ or METTL3 ${ }^{\text {N477A }}$.

866 e: Western blot showing METTL3 protein levels in parental, Mettl3 KO and rescued cell lines with METTL3 $3^{\mathrm{WT}}$, METTL3 ${ }^{\mathrm{APPA}}$, METTL3 ${ }^{\mathrm{W} 475 \mathrm{~A}}$ or METTL3 ${ }^{\mathrm{N} 477 \mathrm{~A}}$.

868 f: Aggregation plots showing METTL3 enrichment levels on IAPEz-int in parental and Mettll4 869 KO cell lines.

$870 \mathrm{~g}$ : Western blots showing METTL14 protein levels in parental and Mettl14 KO cell lines.

871 h: Aggregation plots showing METTL3 enrichment levels on IAPEz-int in parental and $872 R b m 15 / 15 b$ DKO cell lines.

873 i: Western blots showing RBM15 and RBM15B protein levels in parental and $R b m 15 / 15 b$ DKO 874 cell lines.

$875 \mathrm{j}$ : Aggregation plots showing METTL3 enrichment levels on IAPEz-int in control and $\mathrm{m}^{6} \mathrm{~A}$ 876 methyltransferase complex components KD cell lines.

$877 \mathrm{k}$ : Western blots showing protein levels of $\mathrm{m}^{6} \mathrm{~A}$ methyltransferase complex components in 878 control and KD cell lines.

879 1: A cartoon illustrating the dCas9-METTL3 tethering assay in Mettl3 KO cell lines.

880 m: Western blot showing Cas9 and METTL3 protein levels upon Dox treatment.

881 n: ChIP-qPCR of Cas9 (Left) and H3K9me3 (Right) on IAPEz and control regions. The mean 882 of three biological replicates \pm s.d. is shown. ${ }^{*} p<0.05, * * p<0.01$, two-sided t-test. Exact $p$ 883 values are provided in the Source Data.

884 Uniquely+randomly mapped ChIP-seq reads were used in panel b, d, f, h, j.

885 For blots, representative of two independent experiments in panel c, e, g, i, k, m. For blots 886 source data, see Supplementary Figure 1.

887 MTA and MaSat used in ChIP-qPCR are examples of repetitive elements unbound by METTL3. 888 
890 a: Venn diagram showing overlaps of the IAPEz elements bound by METTL3, SETDB1 and 891 TRIM28.

892 b: Scatter plots showing correlation of METTL3 and SETDB1 (Left) or TRIM28 (Right) on 893 IAPEz-int elements ( $\mathrm{n}=2,542)$. Two-sided Pearson's correlation test.

894 c: Boxplots showing SETDB1 (Left) and TRIM28 (Right) enrichment levels on the IAPEz-int 895 elements in parental and Mettl3 KO cell lines. $p=0$ (Left), $p=0$ (Right), two-sided paired t-test. 896 d: ChIP-qPCR showing binding patterns of SETDB1 (Left) and TRIM28 (Right) on IAPEz-int 897 elements in parental and Mettl3 KO cells. The mean of three biological replicates \pm s.d. is 898 shown. $* p<0.05, * * p<0.01$, two-sided t-test. Exact $p$ values are provided in the Source Data. 899 e-f: Boxplots showing density fold changes ( $\log 2($ Mettl3 KO/Parental)) of SETDB1 (e) and 900 TRIM28 (f) on different types of repetitive elements upon Mettl3 KO.

901 g: Co-immunoprecipitation coupled western blot showing interactions of SETDB1 (Left) and 902 TRIM28 (Right) with reintroduced METTL3 (wildtype or catalytically mutated) in Mettl3 KO 903 cells with or without Triptolide treatment.

904 Uniquely mapped ChIP-seq reads were used in panel b, c, e, f.

905 For the boxplots in panel c, e and $\mathrm{f}$, the middle line, lower and upper hinge of the boxplot 906 correspond to the median, the first and third quartiles, respectively. The whiskers extend from 907 the hinges to no further than $1.5 \times$ IQR (inter-quartile range) from the hinge. Outlying points 908 that are plotted individually.

909 For blots, representative of two independent experiments in panel g. For blots source data, see 910 Supplementary Figure 1. 


\section{Extended Data Fig. 6. $\mathbf{m}^{\mathbf{6}}$ A exists on IAPEz-int transcripts}

913 a: RT-qPCR showing relative levels of IAPEz and control RNAs including Actin and Gapdh in 914 different subcellular populations. The mean of three biological replicates \pm s.d. is shown.

915 b: Aggregation plot showing IAPEz-int ChIRP signals enriched on the IAPEz-int elements in 916 the genome.

917 c: Aggregation plot showing in situ ligated DNA of IAPEz-int transcripts revealed by GRID918 seq (GSE82312) enriched on the IAPEz-int elements in the genome.

919 d: Consensus motif of $\mathrm{m}^{6} \mathrm{~A}$ enriched sites (chromatin ribominus RNA).

920 e: Aggregation plot showing the average enrichment levels of $\mathrm{m}^{6} \mathrm{~A}\left(\log 2\left(\mathrm{~m}^{6} \mathrm{~A} / \mathrm{input}\right)\right)$ over 921 coding genes in parental and Mettl3 KO cell lines (chromatin ribominus RNA).

922 f: UCSC snapshots showing $\mathrm{m}^{6} \mathrm{~A}$ enrichment at the 3' end of coding genes, which is depleted 923 in the Mettl3 KO cell lines.

924 g: Western blots showing SETDB1 protein levels in parental and Setdb1 CKO cell lines.

925 h: UCSC snapshots showing $\mathrm{m}^{6} \mathrm{~A}$ enrichment at the 5' end of IAPEz-int, which is depleted in 926 the Mettl3 KO cells.

927 i: In vitro methyltransferase activity of the METTL3/METTL14 with 20-nucleotide RNA 928 substrates containing four repeats of the consensus sequence. The mean of three biological 929 replicates \pm s.d. is shown.

930 Uniquely mapped MeRIP-seq reads were used in panel d, e, f, h. Uniquely+Randomly mapped 931 ChIRP-seq reads and GRID-seq reads were used in panel b, $c$.

932 For blots, representative of two independent experiments in panel g. For blots source data, see 933 Supplementary Figure 1. 
Extended Data Fig. 7. YTHDC1's recruitment to IAPEz chromatin depends on its $\mathbf{m}^{6} \mathrm{~A}$ recognition ability

937 a: ChIP-qPCR showing enrichment levels of nuclear localized $\mathrm{m}^{6} \mathrm{~A}$ reader proteins. The mean

938 of three biological replicates \pm s.d. is shown.

939 b: Venn diagram showing overlaps between METTL3 and YTHDC1 binding events.

940 c: Scatter plot showing correlation of METTL3 and YTHDC1 on IAPEz-int elements.

941 d-e: UCSC snapshot (d) and ChIP-qPCR (e) showing YTHDC1 enrichment on the IAPEz-int

942 elements in parental, Mettl3 KO and rescued cell lines with wildtype or catalytically mutated

943 METTL3. The mean of three biological replicates \pm s.d. is shown. ${ }^{*} p<0.05,{ }^{* *} p<0.01$, two-

944 sided t-test. Exact $p$ values are provided in the Source Data.

945 f: Construction of YTHDC1 $1^{\text {WT }}$, Ythdc1 KO, and YTHDC1 ${ }^{\text {W429A }}$ cell lines using an auxin-

946 inducible degron (AID) system.

947 g: Western blots showing IAA-induced rapid degradation of AID-YTHDC1.

948 h-k: Heatmaps (h), UCSC snapshots (i), Boxplots (j) and Aggregation plots (k) showing 949 YTHDC1 levels on IAPEz-int in YTHDC1 ${ }^{\mathrm{WT}}$, Ythdc1 KO, and YTHDC1 ${ }^{\mathrm{W} 429 \mathrm{~A}}$ cell lines. **** $950 p<0.0001$ (Exact $p$ values from left to Right:0, 0), two-sided paired t-test.

951 1: ChIP-qPCR showing YTHDC1 enrichment levels on the IAPEz-int elements in control

$952 \mathrm{mESCs}$ and mESCs treated with $\alpha$-amanitin, flavopiridol and triptolide, respectively. The mean 953 of three biological replicates \pm s.d. is shown. ${ }^{*} p<0.05, * * p<0.01$. two-sided t-test. Exact $p$ 954 values are provided in the Source Data.

955 Uniquely mapped ChIP-seq reads were used in panel b, c, d, h, i, j. Uniquely+randomly mapped 956 ChIP-seq reads were used in panel $\mathrm{k}$.

957 Heatmaps were ranked according to METTL3 density in parental cells in descending order in 958 panel h.

959 For the boxplots in $\mathrm{j}$, the middle line, lower and upper hinge of the boxplot correspond to the 960 median, the first and third quartiles, respectively. The whiskers extend from the hinges to no 961 further than $1.5 \times$ IQR (inter-quartile range) from the hinge. Outlying points that are plotted 962 individually.

963 For blots, representative of two independent experiments in panel g. For blots source data, see 964 Supplementary Figure 1.

965 MTA and MaSat used in ChIP-qPCR are examples of repetitive elements unbound by METTL3. 966 

a-c: Boxplots (a), and Aggregation plots (b), and UCSC snapshot (c) showing METTL3 levels on IAPEz-int in YTHDC1 $1^{\mathrm{WT}}$, Ythdc1 KO and YTHDC1 ${ }^{\mathrm{W} 429 \mathrm{~A}}$ cell lines. $* * * * p<0.0001$ (Exact $p$ values from left to Right: $2.3 \mathrm{e}-176,1.2 \mathrm{e}-195)$, two-sided paired t-test. d-f: Boxplots (d), and Aggregation plots (e), and UCSC snapshot (f) showing H3K9me3 levels on IAPEz-int in YTHDC $1^{\mathrm{WT}}$, Ythdc1 KO and YTHDC1 ${ }^{\mathrm{W} 429 \mathrm{~A}}$ cell lines. ${ }^{* * * *} \mathrm{p}<0.0001$ (Exact $p$ values from left to Right: 7.1e-63, 4.8e-279), two-sided paired t-test. g-j: Heatmaps (g), and boxplots (h), and Aggregation plots (i), and UCSC snapshots (j) showing H4K20me3 levels on IAPEz-int in YTHDC $1^{\mathrm{WT}}$, Ythdc1 KO and YTHDC $1^{\mathrm{W} 429 \mathrm{~A}}$ cell lines. **** $\mathrm{P}<0.0001$ (Exact $p$ values from left to Right: $8.8 \mathrm{e}-30,7.8 \mathrm{e}-114$ ), two-sided paired t-test. $\mathrm{k}$ : Western blots showing protein levels of METTL3 and YTHDC1 in control, Mettl3 KO, Ythdc1 KD and Mettl3 KO+Ythdc1 KD cell lines. 1: ChIP-qPCR showing H3K9me3 enrichment level on METTL3 elements in control, Mettl3 $\mathrm{KO}$, Ythdc1 KD and Mettl3 KO+Ythdc1 KD cell lines. ${ }^{*} p<0.05$, ** $p<0.01$. two-sided t-test. Exact $p$ values are provided in the Source Data. m: Western blots showing Cas9 protein levels upon Dox treatment in Mettl3 KO cell lines. n: ChIP-qPCR of H3K9me3 (Left) and Cas9 (Right) on IAP and control regions in Mettl3 KO cell lines expressing dCas9-YTHDC1. The mean of three biological replicates \pm s.d. is shown. $* p<0.05, * * p<0.01$. two-sided t-test. Exact $p$ values are provided in the Source Data. o: Western blots showing Cas9 and METTL3 protein levels upon Dox treatment in Mettl3 $\mathrm{KO}+$ METTL $3^{\text {APPA }}$ cell lines.

988 p: ChIP-qPCR of H3K9me3 (Left) and Cas9 (Right) on IAP and control in Mettl3 989 KO+METTL3 ${ }^{\text {APPA }}$ cell lines expressing dCas9-YTHDC1. The mean of three biological replicates \pm s.d. is shown. Exact $p$ values are provided in the Source Data. q: Co-immunoprecipitation coupled with western blots showing interactions of YTHDC1 with reintroduced METTL3 (wildtype or catalytically mutated) in Mettl3 KO cells with or without Triptolide treatment. mapped ChIP-seq reads were used in panel b, e, i.

996 Heatmaps were ranked according to METTL3 density in parental cells in descending order in panel g.

998 For the boxplots in panel a, d, h, the middle line, lower and upper hinge of the boxplot correspond to the median, the first and third quartiles, respectively. The whiskers extend from the hinges to no further than $1.5 \times \mathrm{IQR}$ (inter-quartile range) from the hinge. Outlying points that are plotted individually.

1002 For blots, representative of two independent experiments in panel k, m, q. For blots source data, see Supplementary Figure 1. MTA and MaSat used in ChIP-qPCR are examples of repetitive elements unbound by METTL3. 


\section{Extended Data Fig. 9. SETDB1 regulates METTL3/ YTHDC1 recruitment}

1007 a-d: Heatmaps (a), UCSC snapshot (b), boxplot (c) and aggregation plots (d) showing METTL3

1008 enrichment levels on IAPEz-int in parental and Setdb1 CKO cells. $p$ value $=1.3 \mathrm{e}-48$, two-sided 1009 paired t-test.

1010 e-h: Heatmaps (e), UCSC snapshot (f), boxplot (g) and aggregation plots (h) showing YTHDC1

1011 enrichment levels on IAPEz-int in parental and Setdb1 CKO cells. $p$ value $=4.5 \mathrm{e}-100$, two-

1012 sided paired t-test.

1013 Uniquely mapped ChIP-seq reads were used in panel a, b, c, e, f, g. Uniquely+randomly 1014 mapped ChIP-seq reads were used in panel $\mathrm{d}, \mathrm{h}$.

1015 Heatmaps were ranked according to METTL3 density in parental cells in descending order in 1016 panel a, e.

1017 For the boxplots in panel c, g, the middle line, lower and upper hinge of the boxplot correspond 1018 to the median, the first and third quartiles, respectively. The whiskers extend from the hinges 1019 to no further than $1.5 \times \mathrm{IQR}$ (inter-quartile range) from the hinge. Outlying points that are 1020 plotted individually. 
1022 Extended Data Fig. 10. RNA dependent heterochromatin formation models

1023 a. RNA dependent heterochromatin formation on IAPEz-int in mESC. Specifically, METTL3 1024 together with other $\mathrm{m}^{6} \mathrm{~A}$ methyltransferase components methylate IAPEz transcripts, which are 1025 recognized by the $\mathrm{m}^{6} \mathrm{~A}$ reader protein YTHDC1. YTHDC1 in turn stabilizes METTL3 binding, 1026 possibly through protein-protein interaction. Chromatin associated METTL3 enhances 1027 SETDB1/TRIM28 binding, which in turn stabilizes METTL3 recruitment.

1028 b. RNA dependent heterochromatin formation on centromere regions in S. pombe. Specifically, 1029 heterochromatin generation over centromere regions is initiated by the base-paring recognition 1030 and binding of RITS complex to the RNAs transcribed from these regions, which in turn 1031 enhances sRNA generation through recruitment of RDRC. RITS then recruits CLRC to 1032 catalyze H3K9 methylation, which in turn promotes RITS binding.

1033 c. RNA dependent heterochromatin formation on DSR genes in S. pombe. Specifically, Mmi1 1034 protein recognizes the DSR consensus motif on the RNAs transcribed from these genes and 1035 then recruits the H3K9 methyltransferase Clr4 through Red1. 
Fig. 1. METTL3 binds endogenous retroviral elements

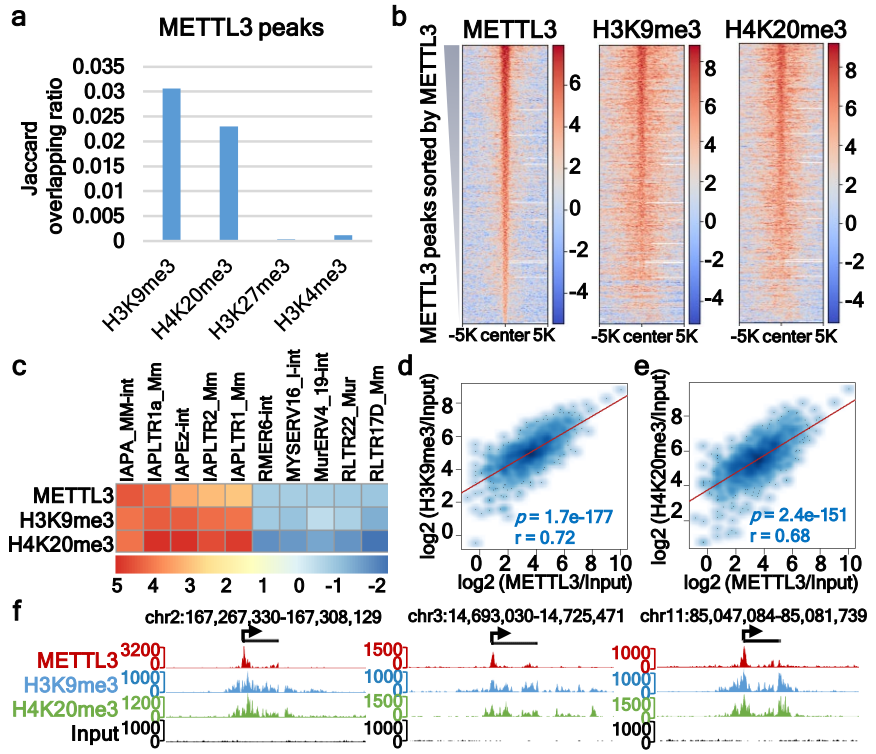


Fig. 2. METTL3 is required for heterochromatin formation over repetitive elements

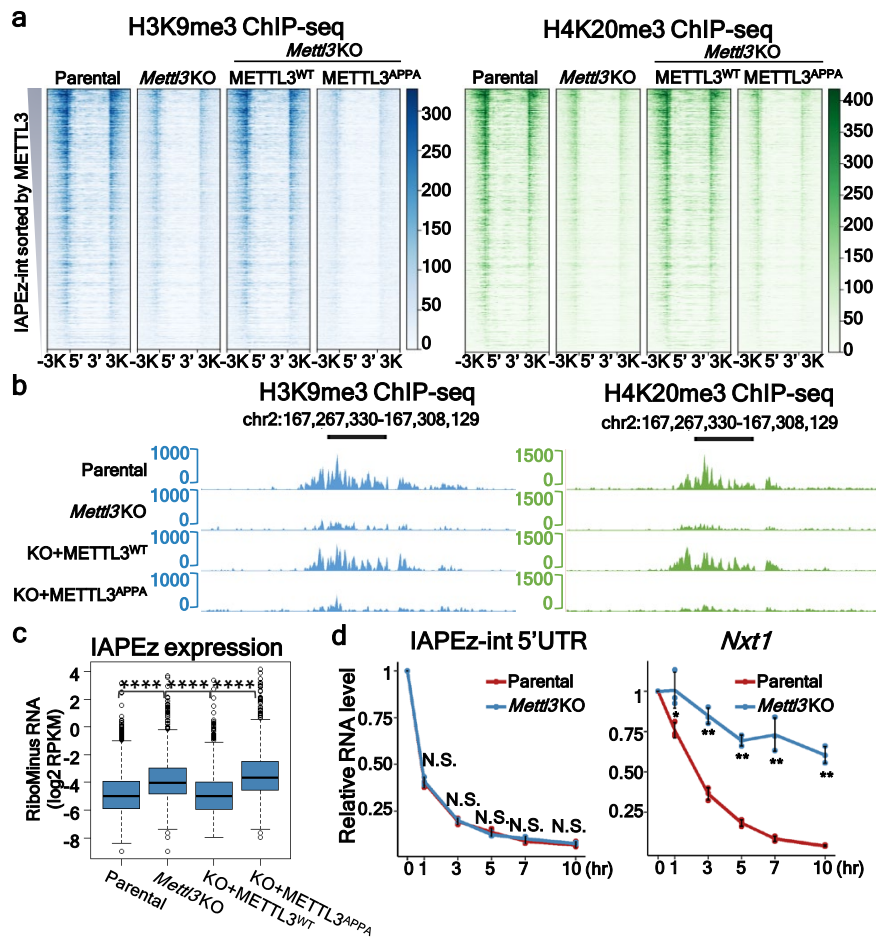


Fig. 3. METTL3 regulates SETDB1/TRIM28 localization to IAPEz elements

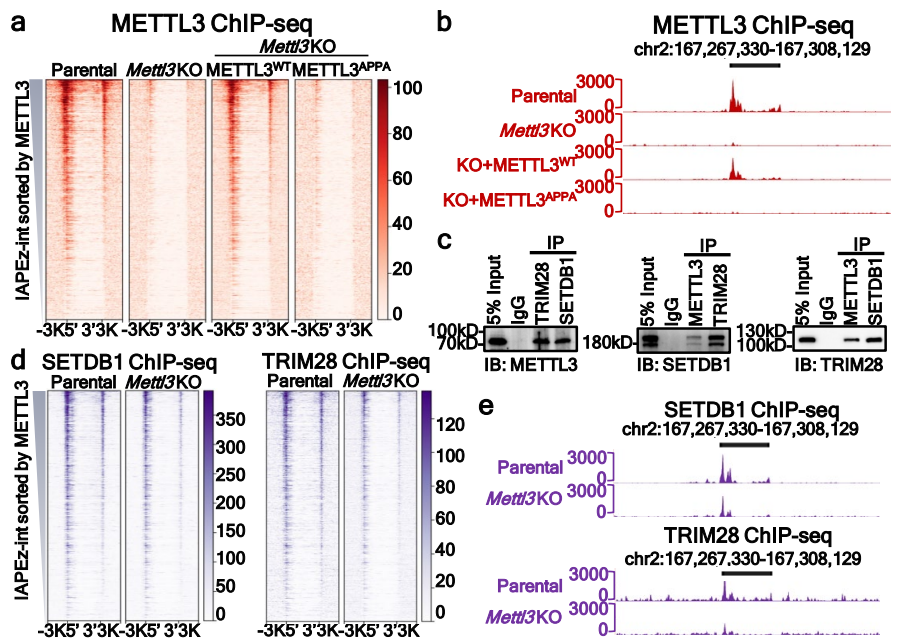


Fig. 4. YTHDC1 recruited by METTL3 dependent m6A contributes to METTL3 binding and heterochromatin formation on IAPEz-int
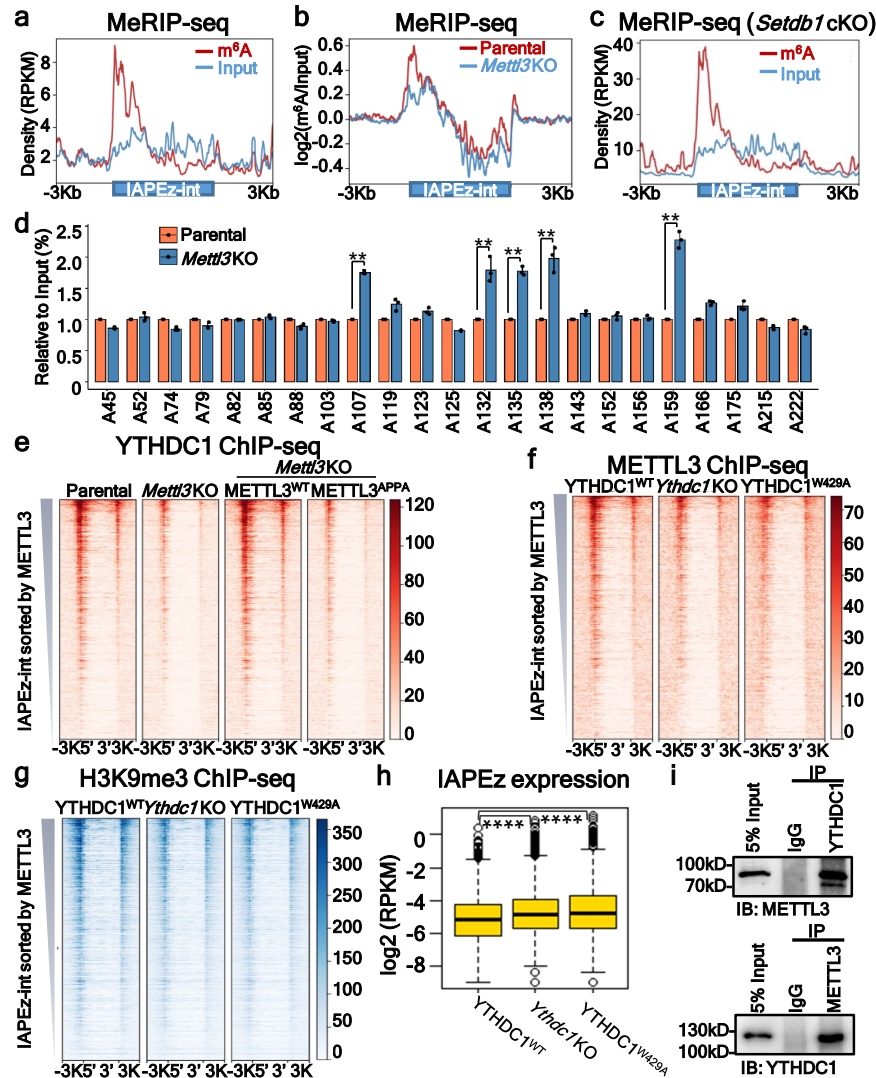
Extended Data Fig. 1. METTL3 binds endogenous retroviral elements
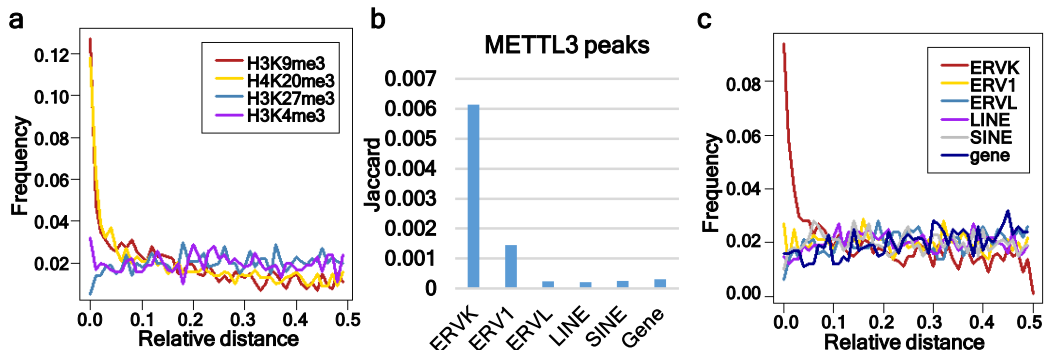

d

e

Correlation on ERVK subtypes

Correlation on ERVK subtypes
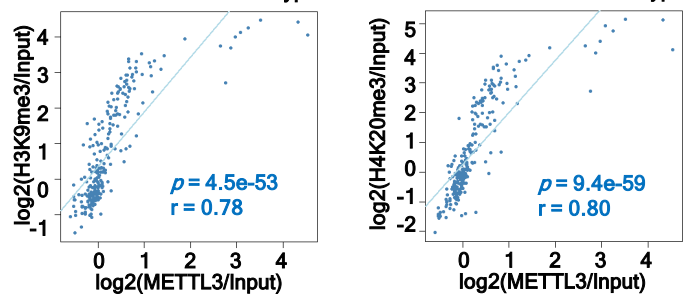

f

g

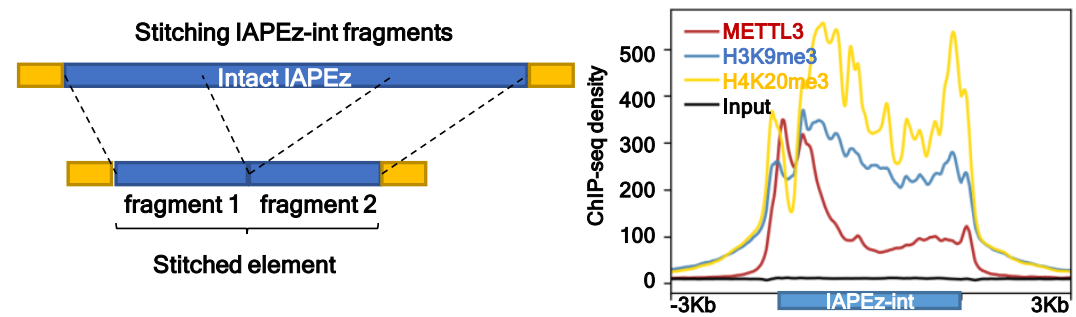

h

METTL3 ChIP-qPCR

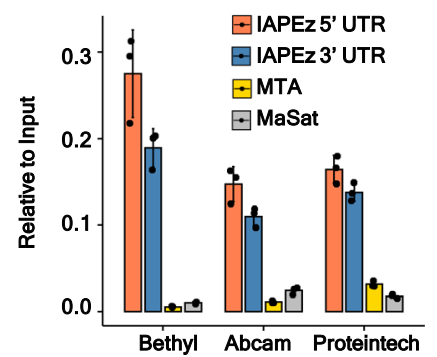

i
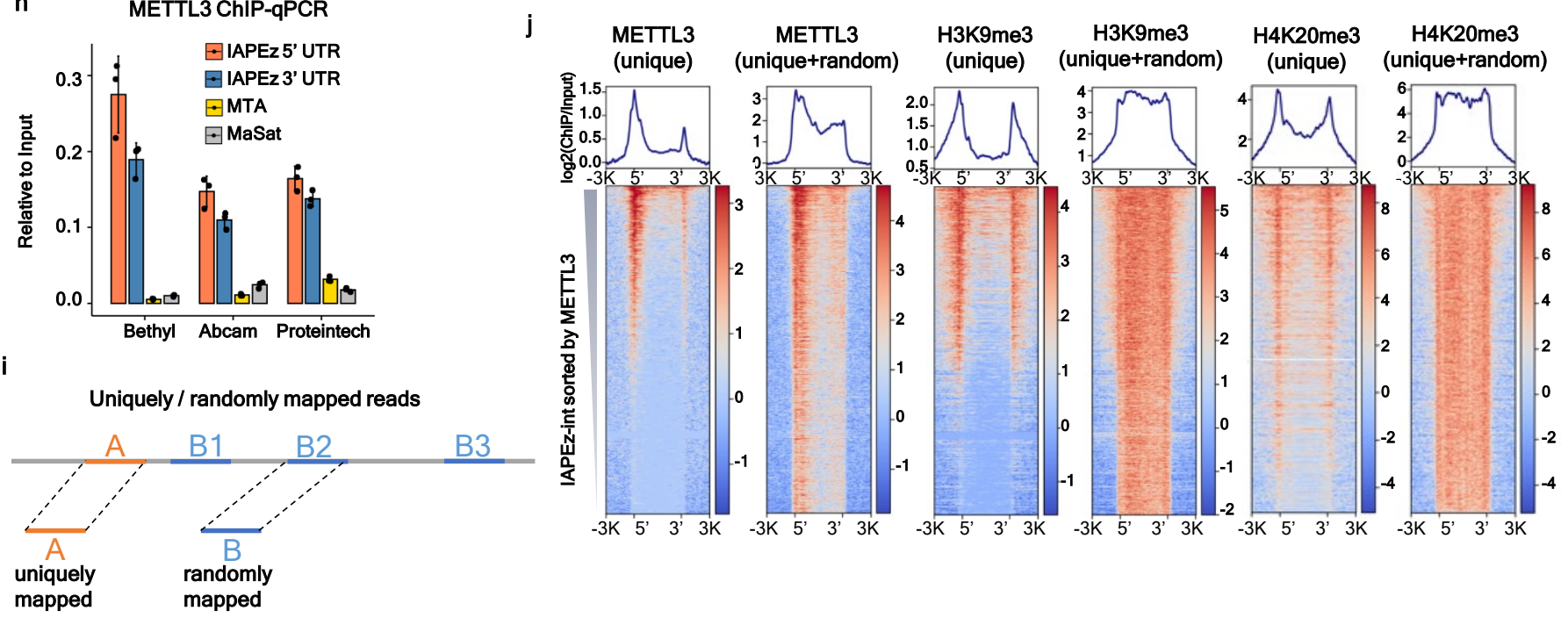
Extended Data Fig. 2. METTL3 is required for heterochromatin formation
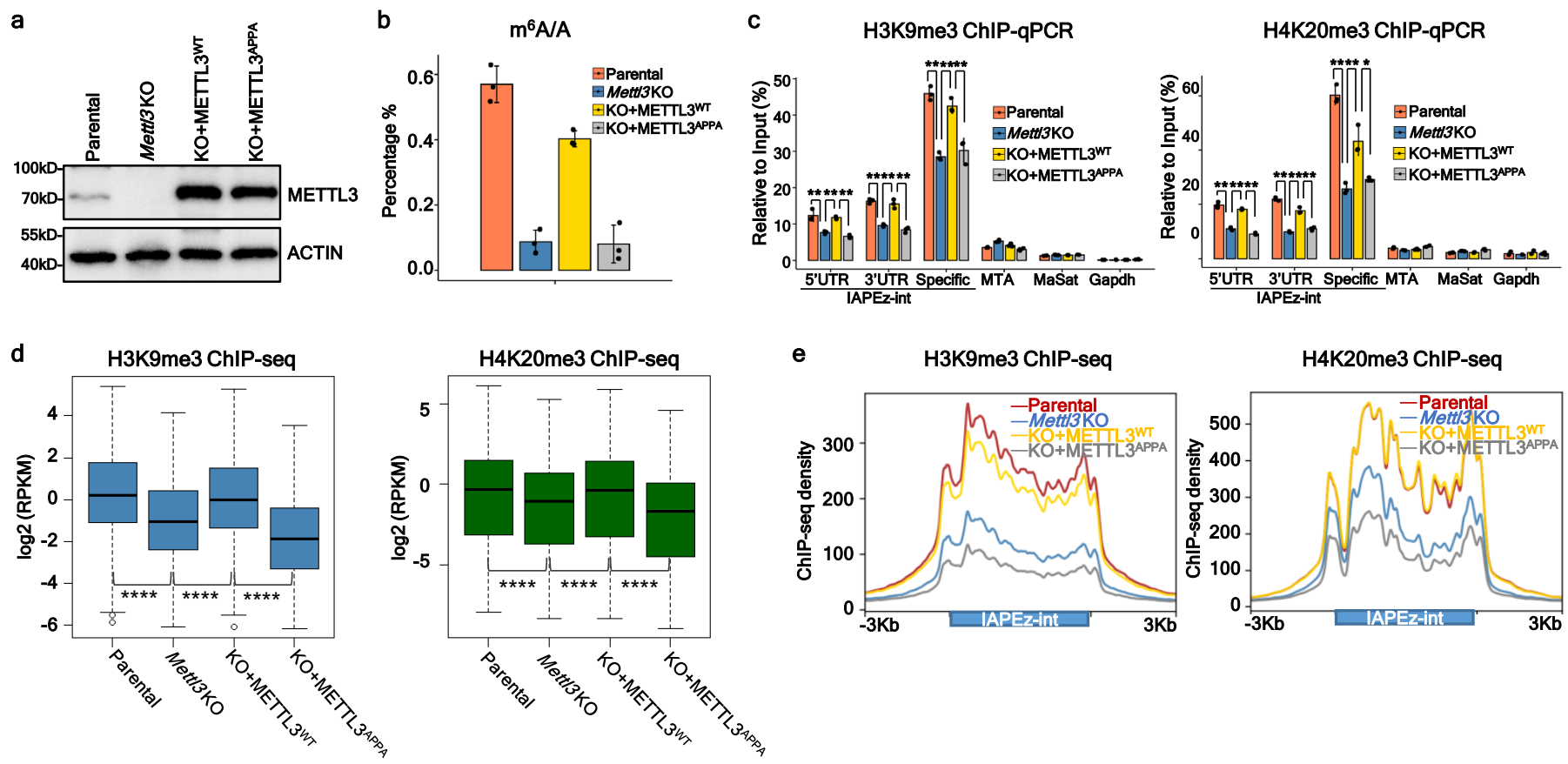

e H3K9me3 ChIP-seq
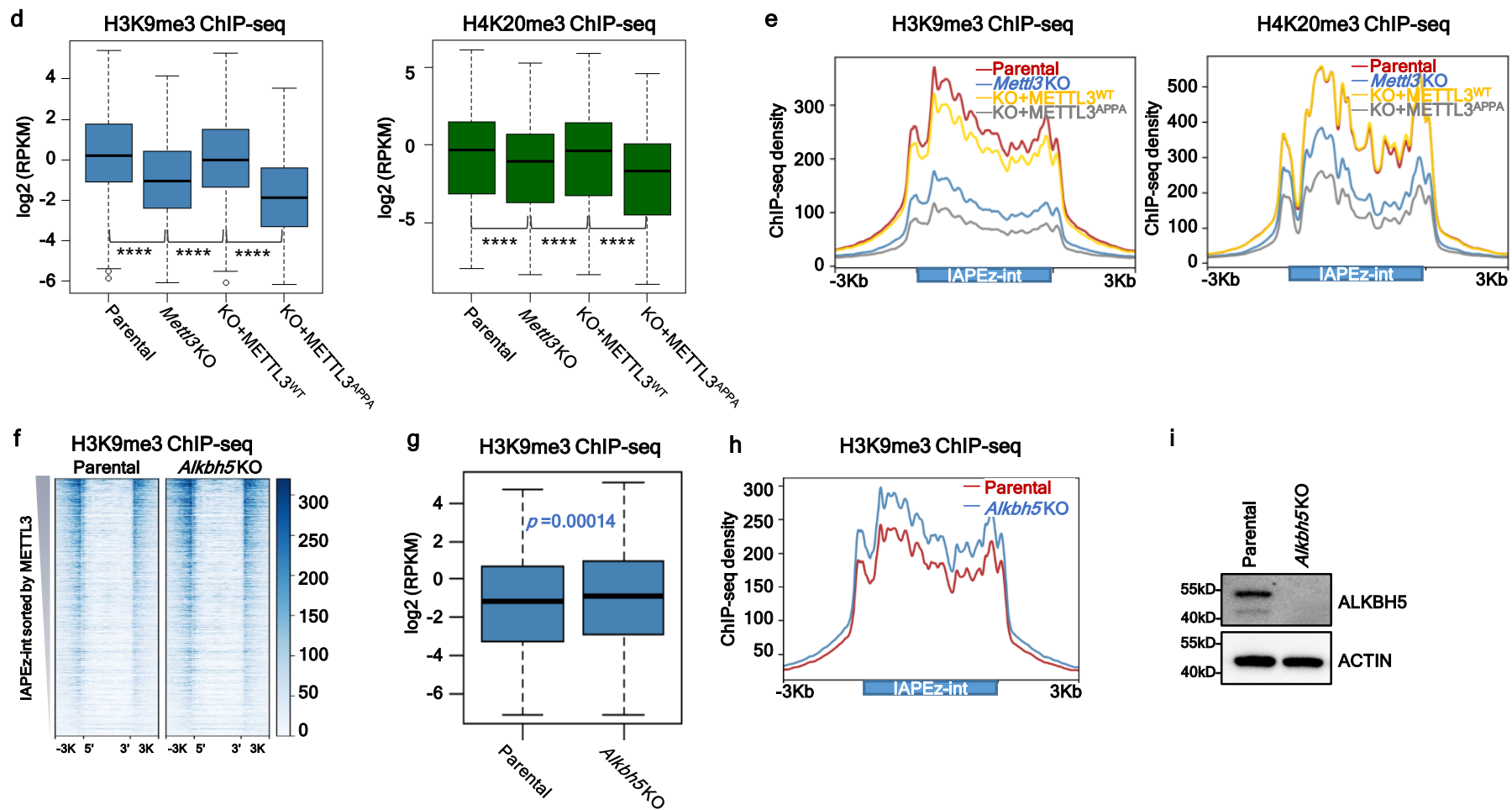

j

H3K9me3 change

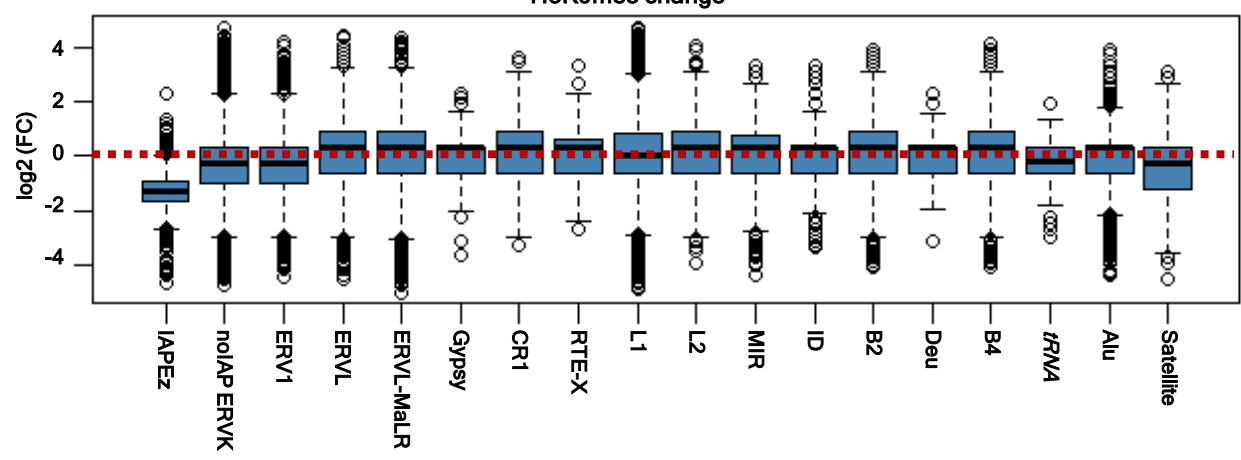

$\mathbf{k}$ H4K20me3 change

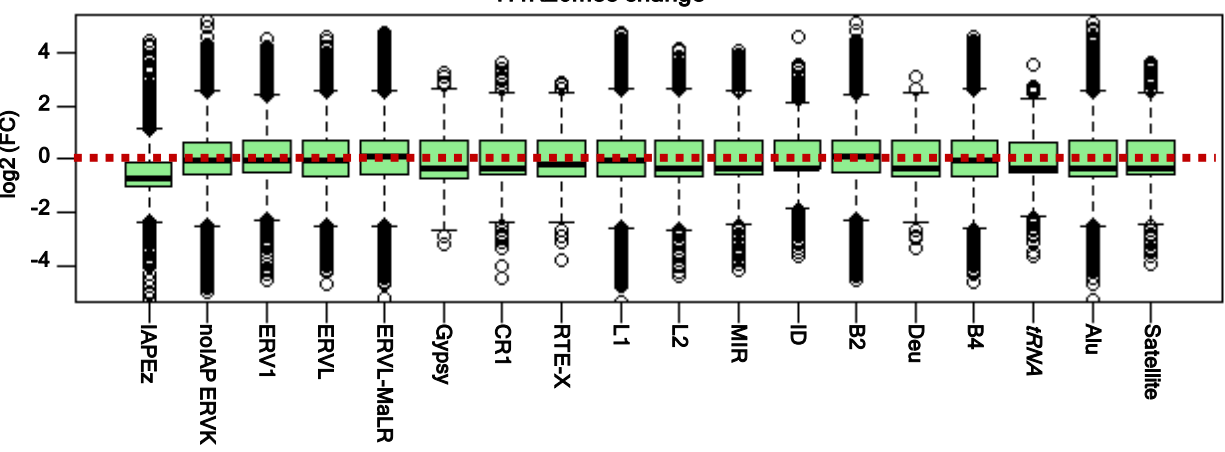


Extended Data Fig. 3. METTL3 is required for heterochromatin formation
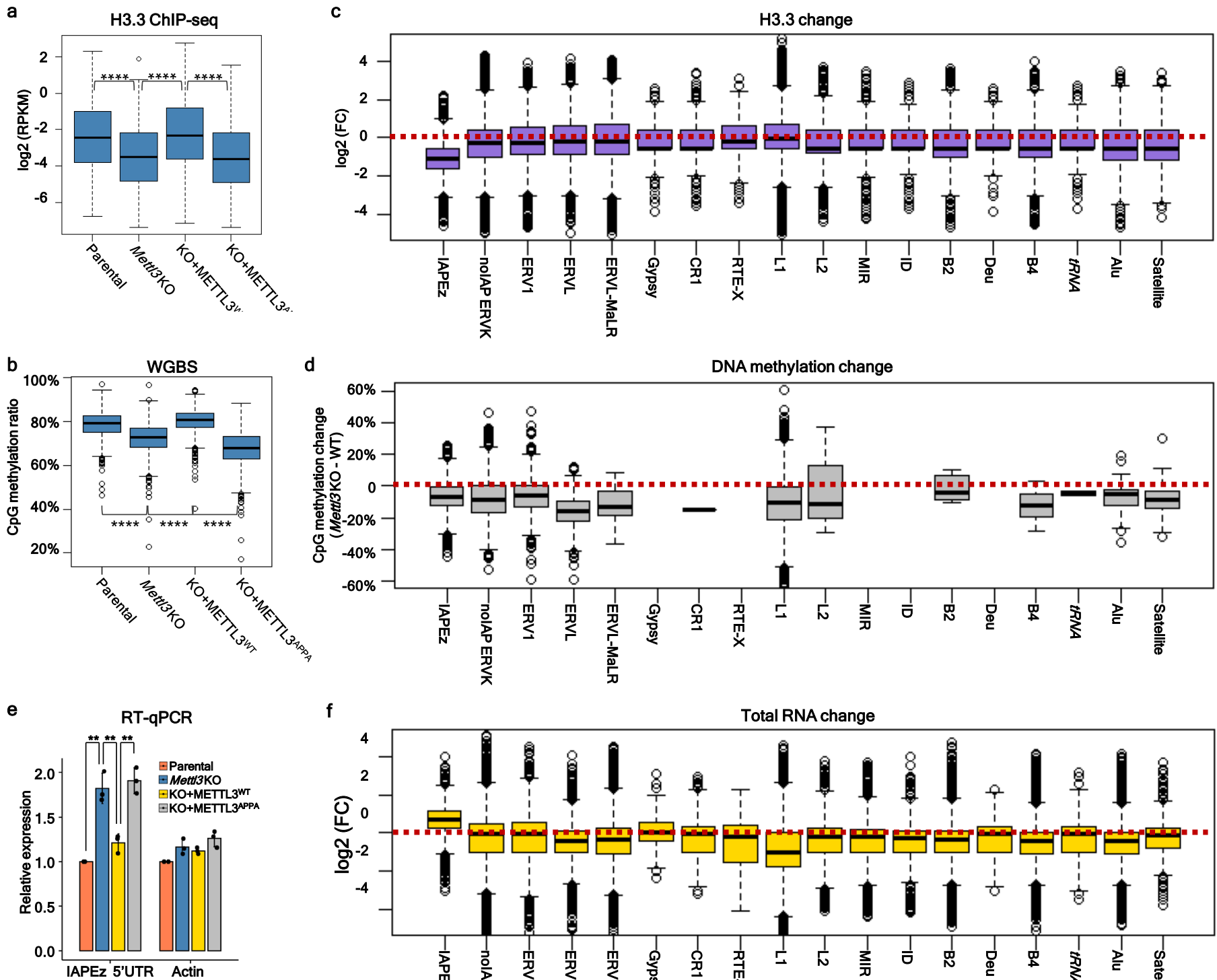

f

Total RNA change

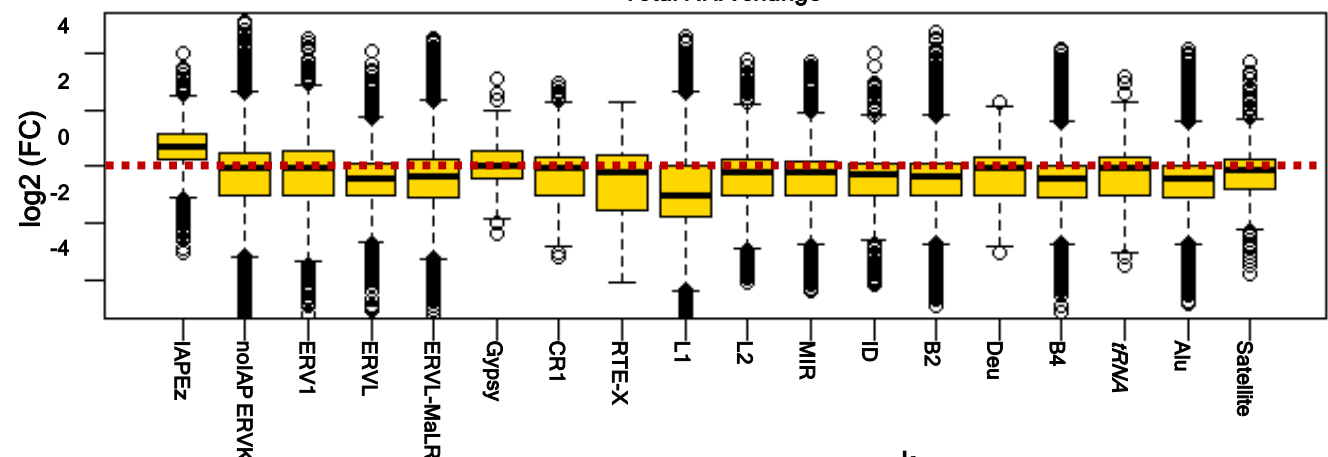

k IAPEz expression
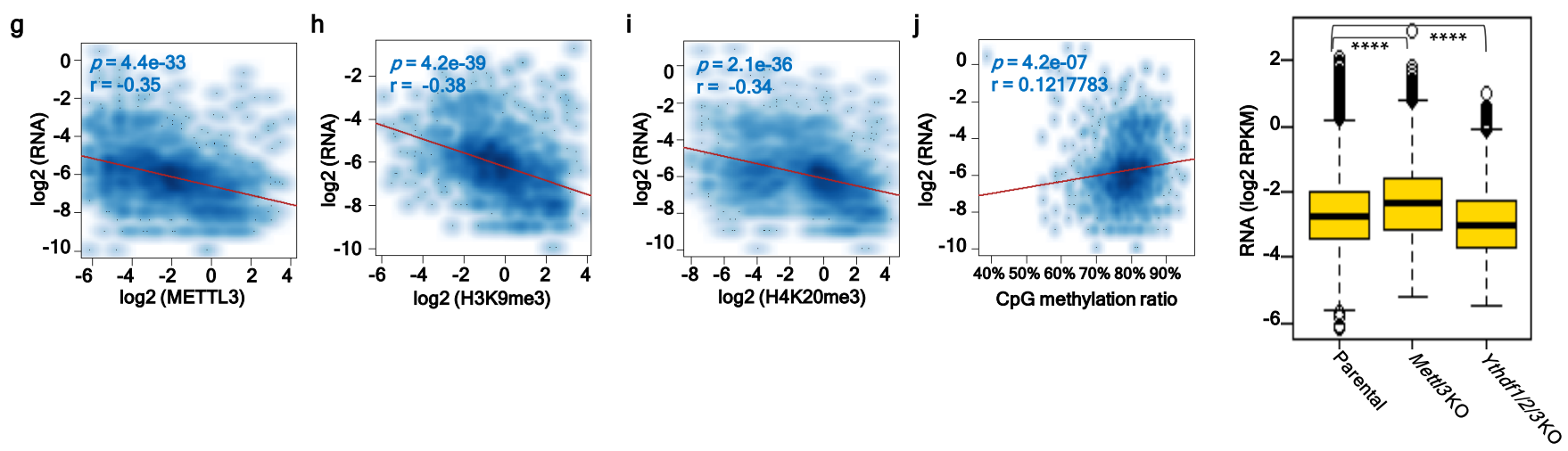
Extended Data Fig. 4. METTL3 chromatin binding is dependent on its own catalytic activity
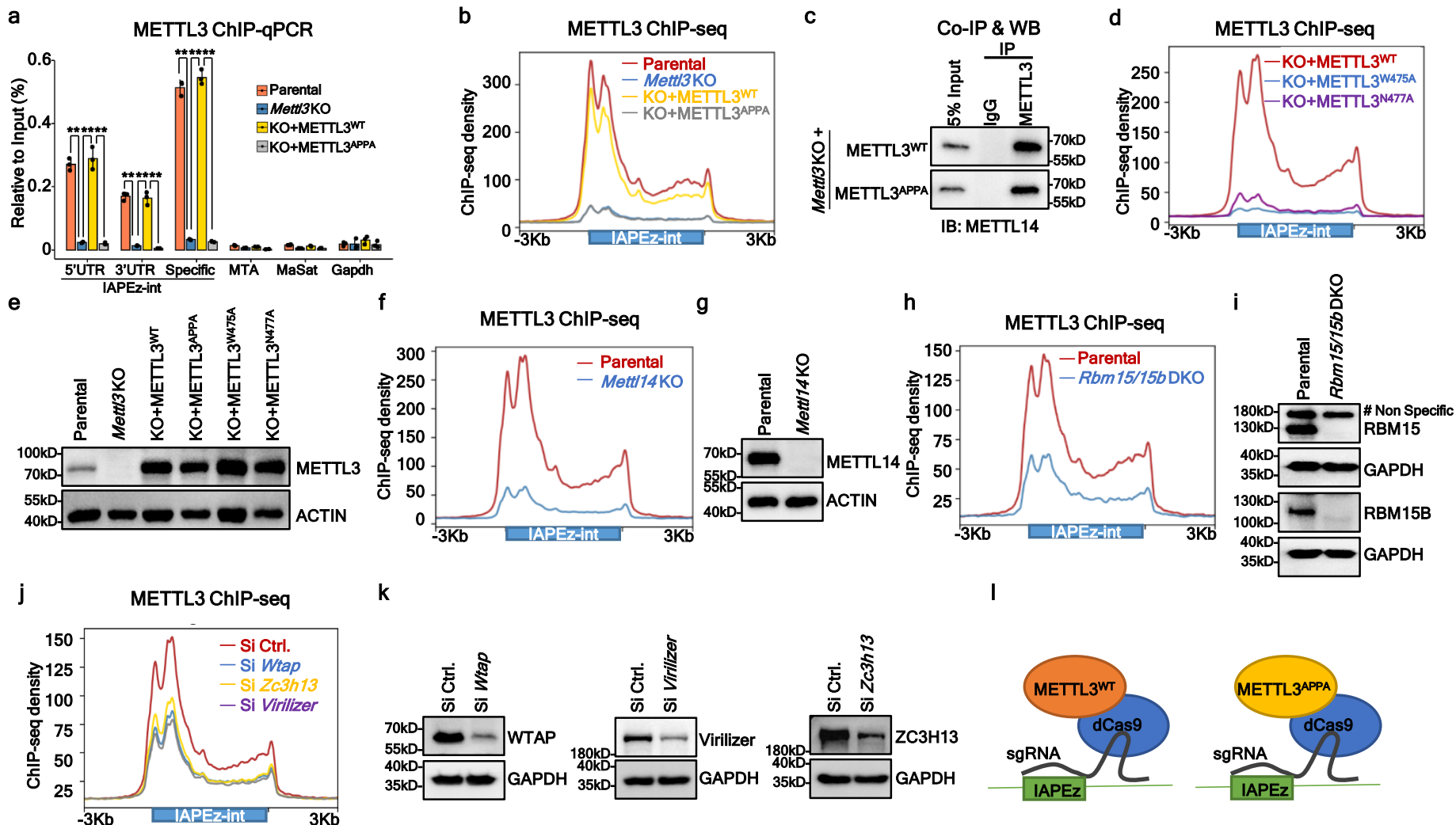

k
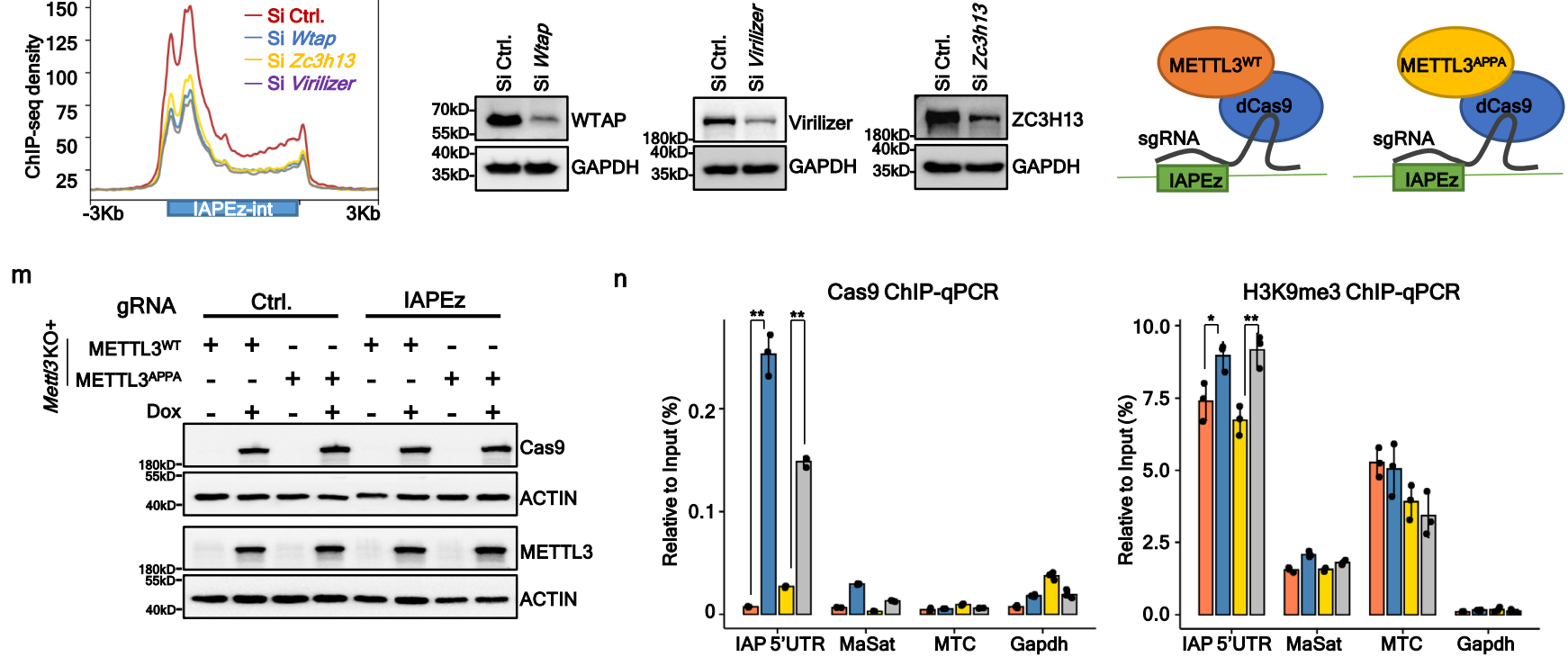

๑Ctrl. gRNA+METTL3WT FIAPEZ gRNA+METTL3WT

- Ctrl. gRNA+METTL3APPA $\rightleftharpoons$ IAPEZ gRNA+METTL3APPA 
Extended Data Fig. 5. METTL3 regulates SETDB1/ TRIM28 recruitment
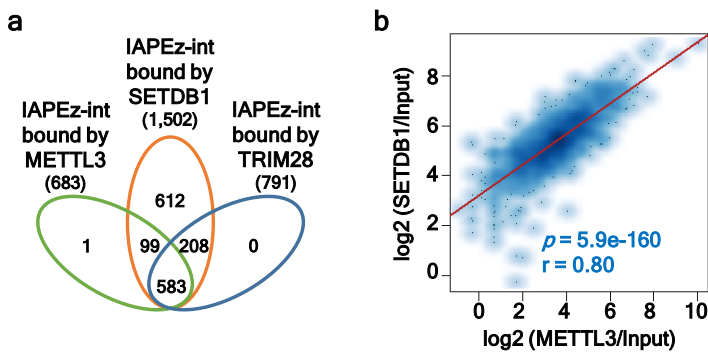

c

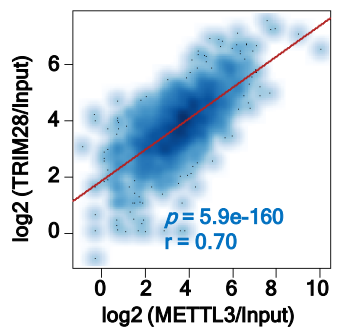

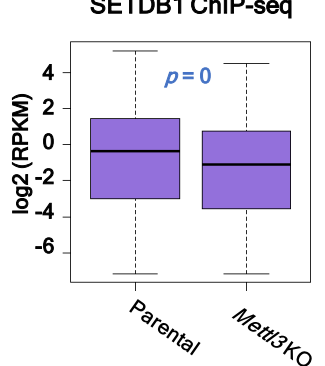

TRIM28 ChIP-seq

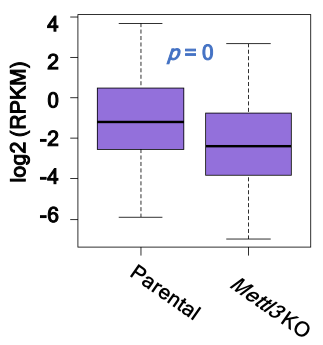

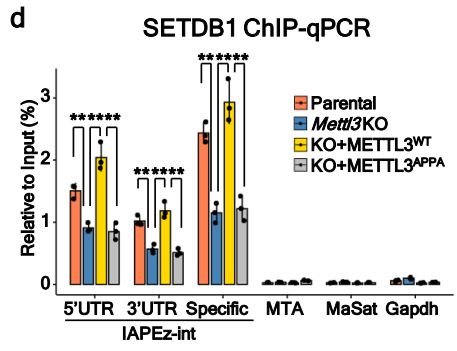

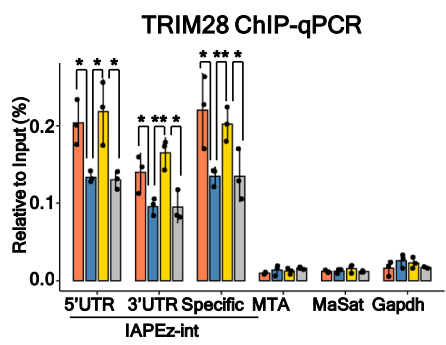

TRIM28 change

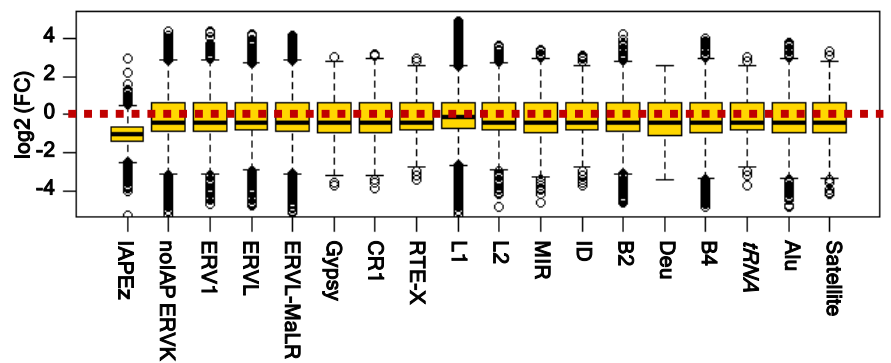

SETDB1 change

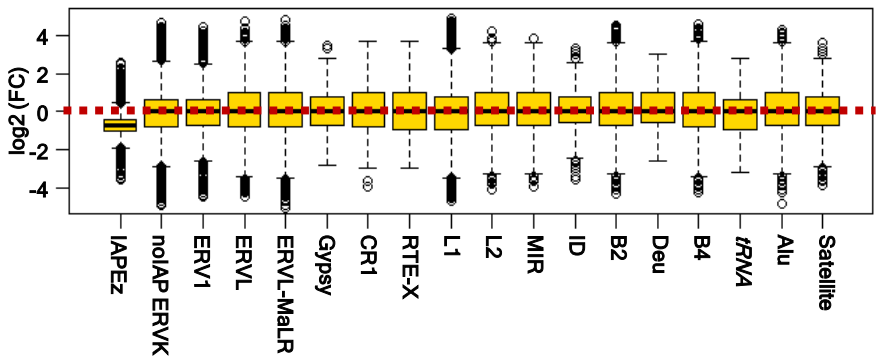

g

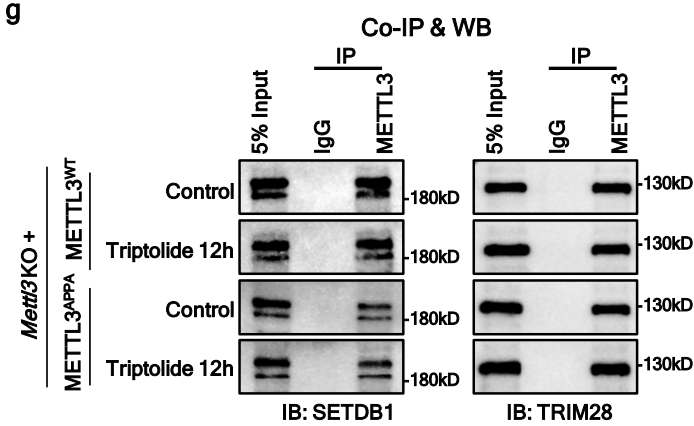


Extended Data Fig. 6. m $^{6} \mathrm{~A}$ exists on IAPEz-int transcripts

a

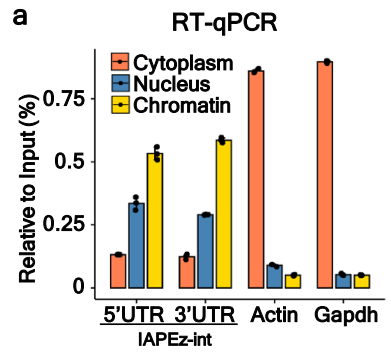

e
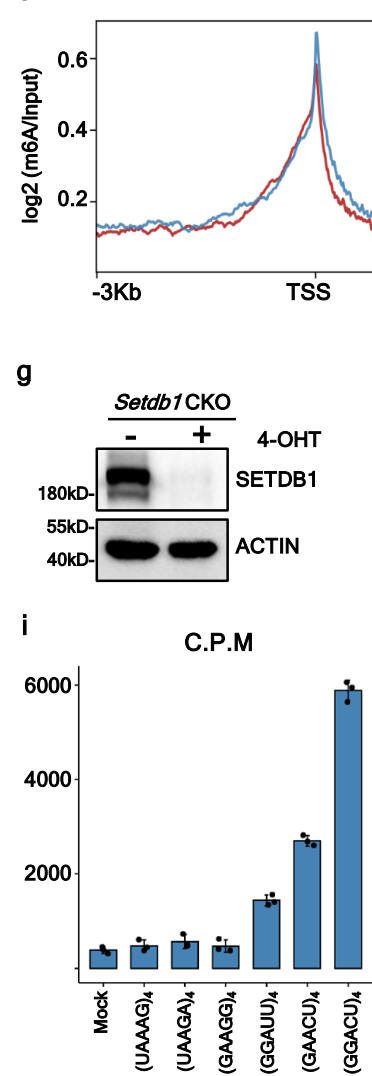

b

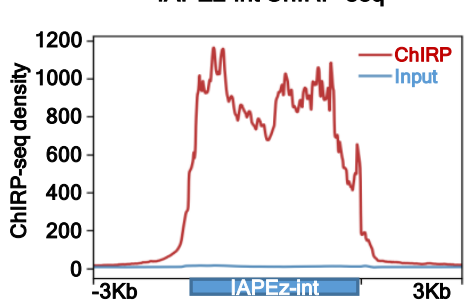

MeRIP-seq

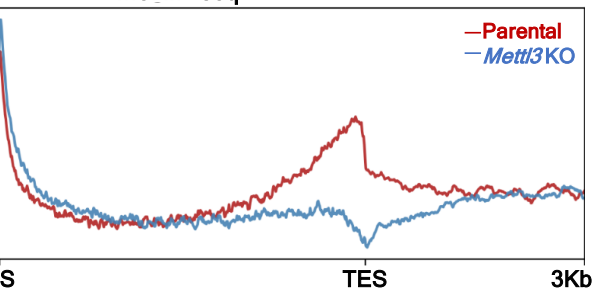

c
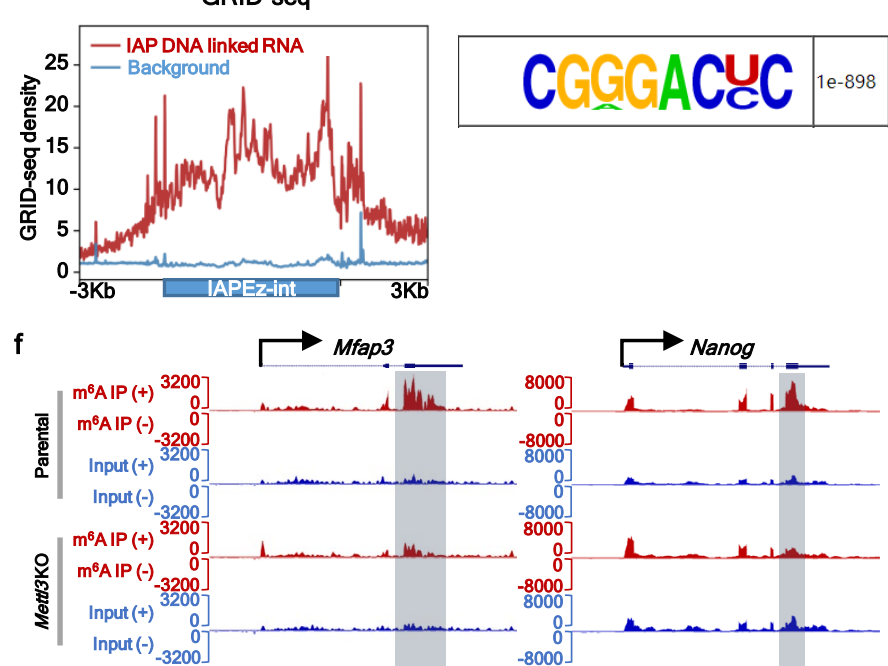

h

chr16:89,440,787-89,450,786

chr16:58,648,819-58,657,018 chrX:96,346,201-96,354,477
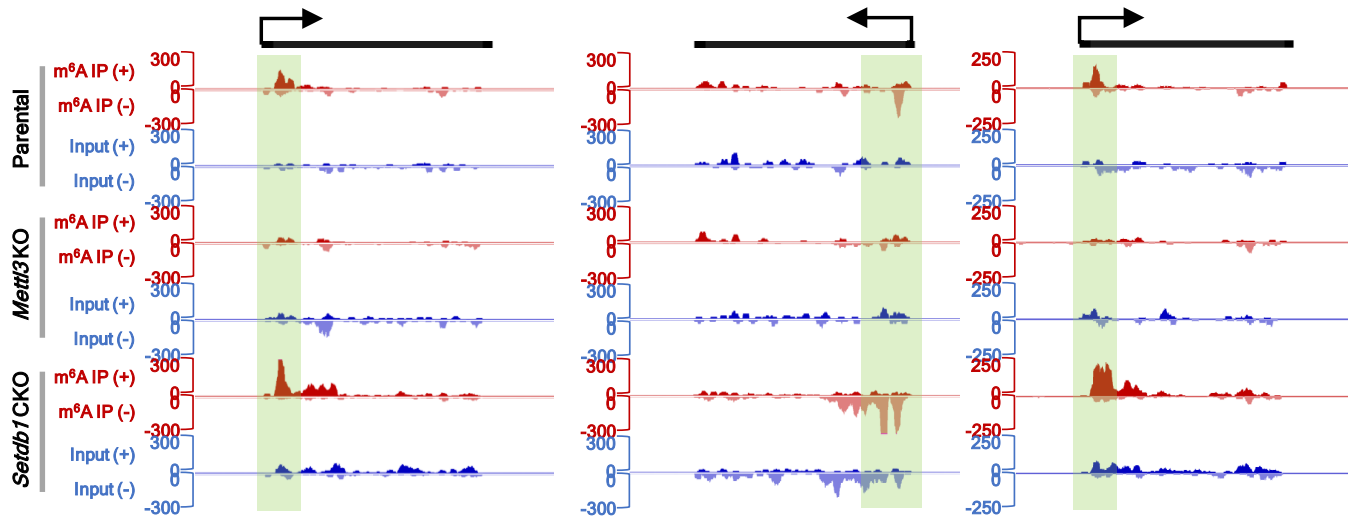
Extended Data Fig. 7. YTHDC1's recruitment to IAPEz chromatin depends on its $\mathrm{m}^{6} \mathrm{~A}$ recognition ability

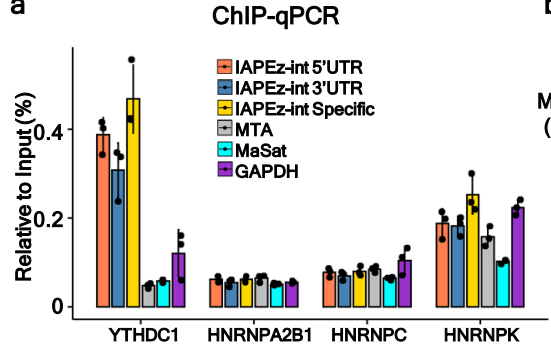

e

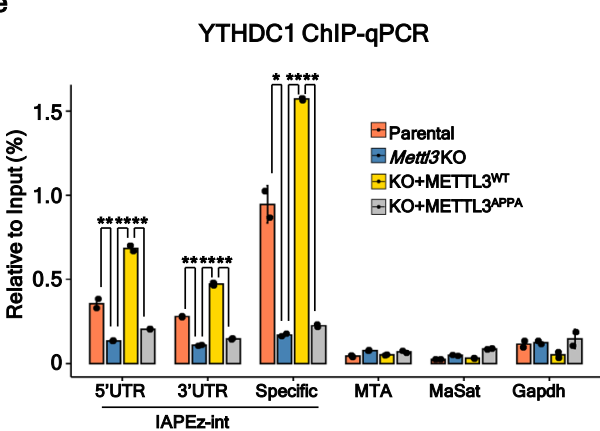

b

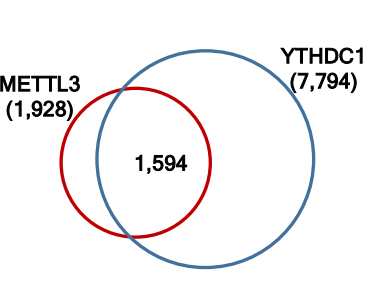

c

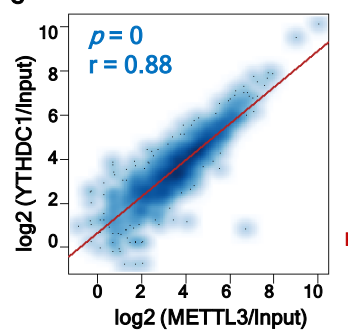

d YTHDC1 ChIP-seq

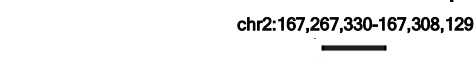

g

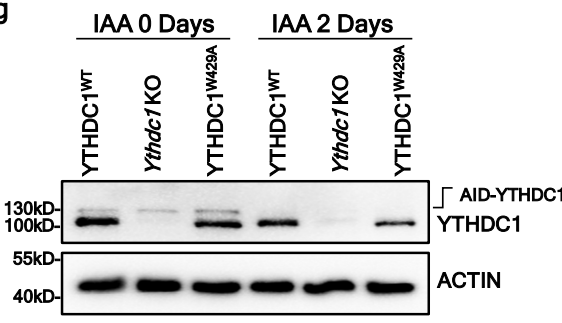

f

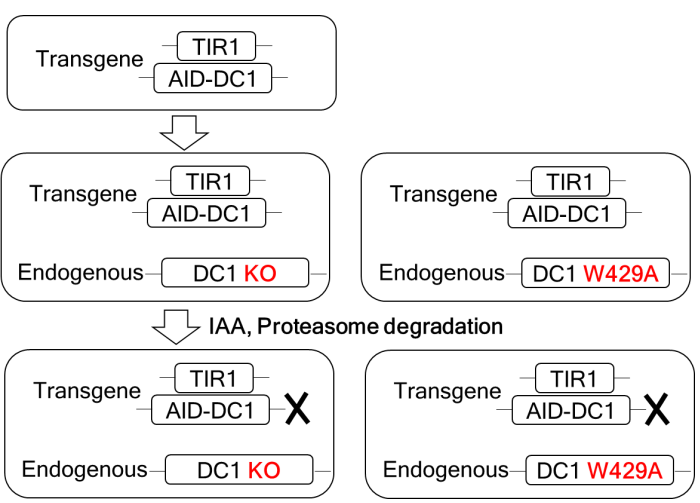

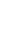

h YTHDC1 ChIP-seq i
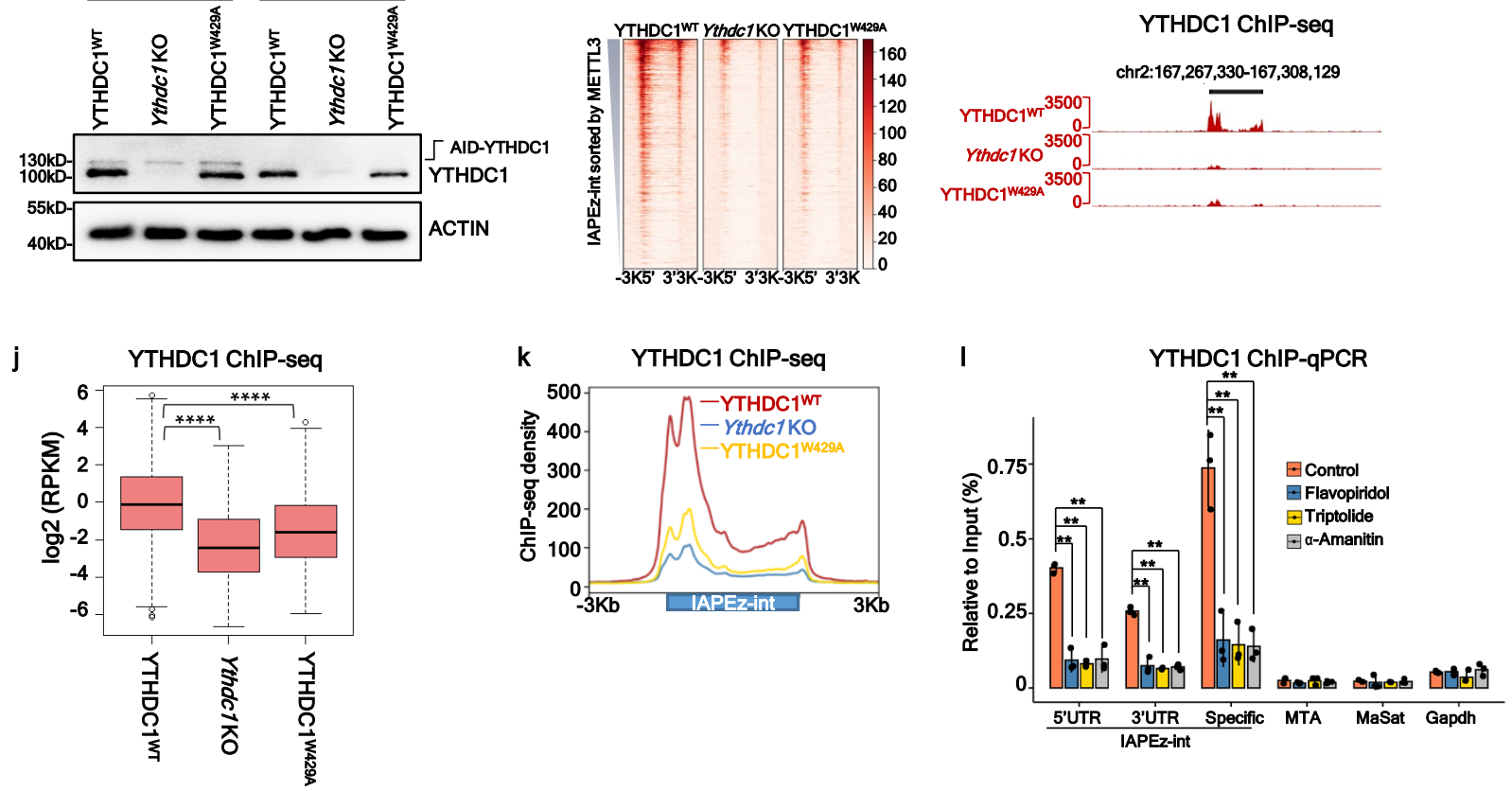
Extended Data Fig. 8. YTHDC1 stabilizes METTL3 on heterochromatin

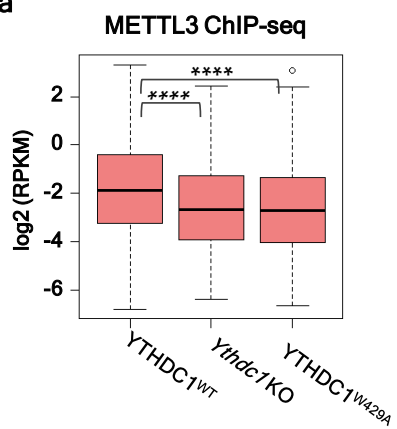

e
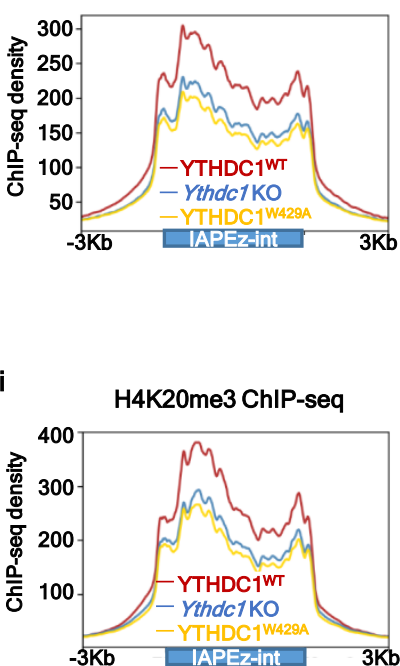

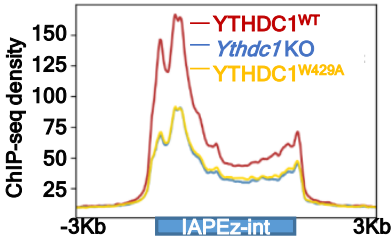

f

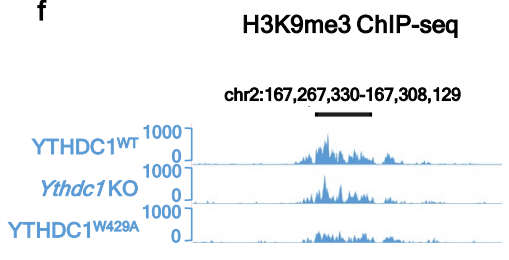

j

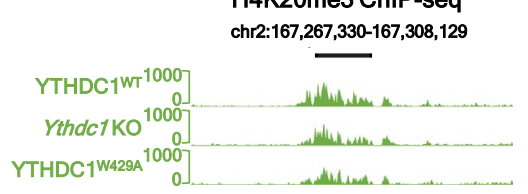

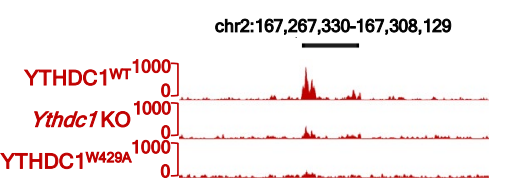

d

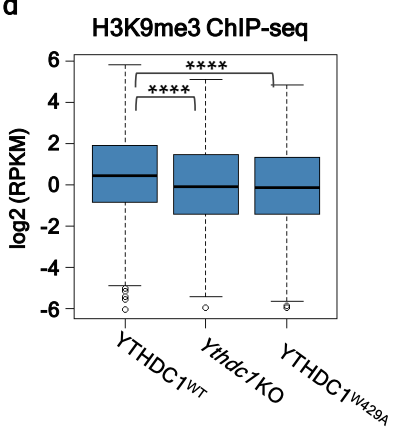

h

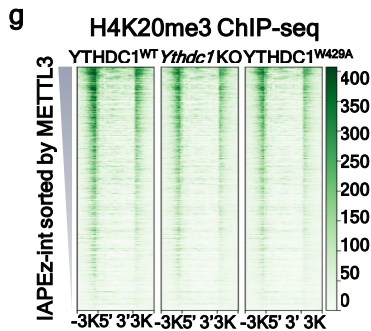

k

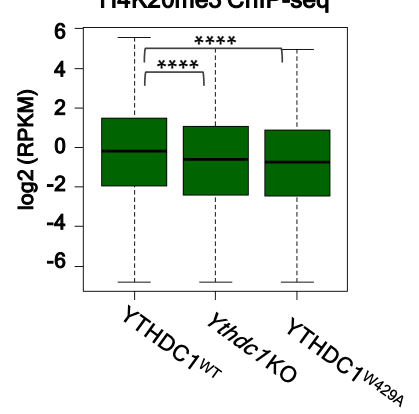

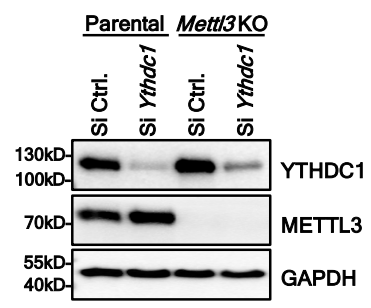

|

m
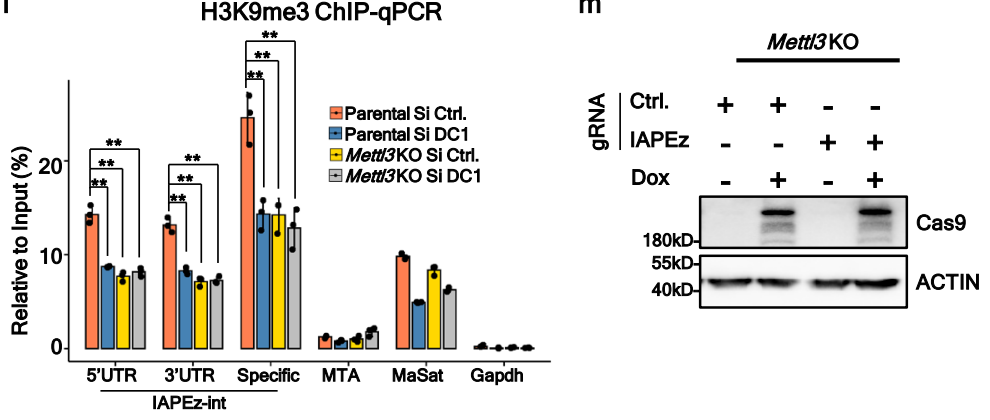

Mett/3KO H3K9me3 ChIP

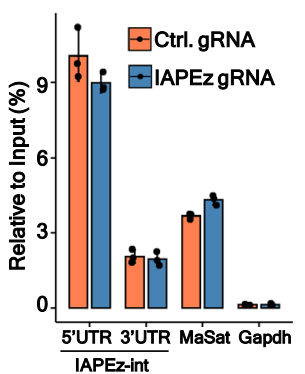

Mett/3KO Cas9 ChIP

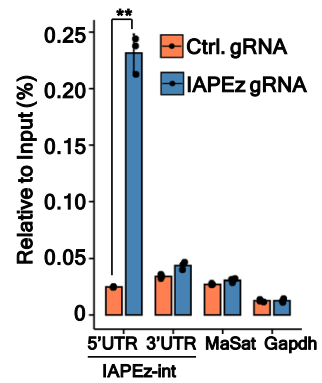

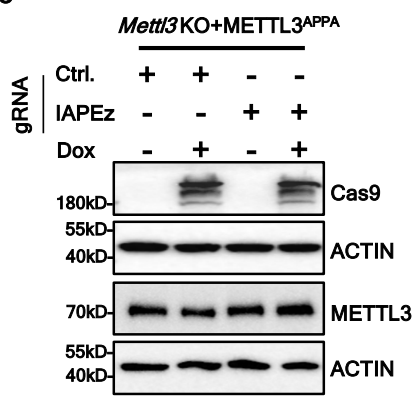

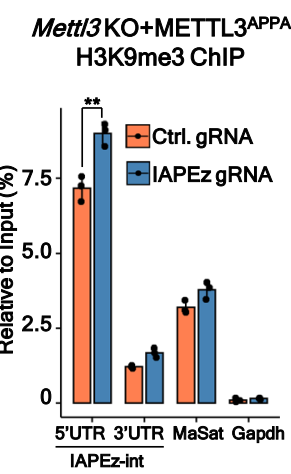

Mett/3KO+METTL3 ${ }^{\text {APPA }}$ Cas9 ChIP

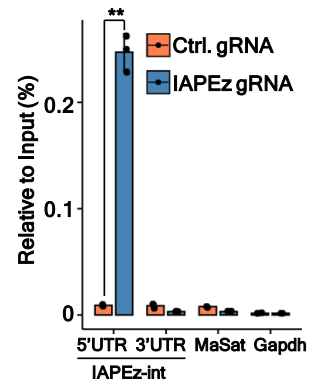

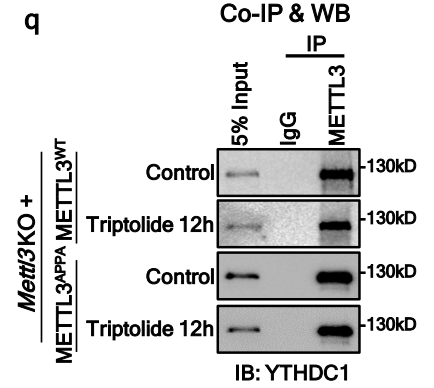

q

IB: YTHDC1 


\section{Extended Data Fig. 9. SETDB1 regulates METTL3/ YTHDC1 recruitment}

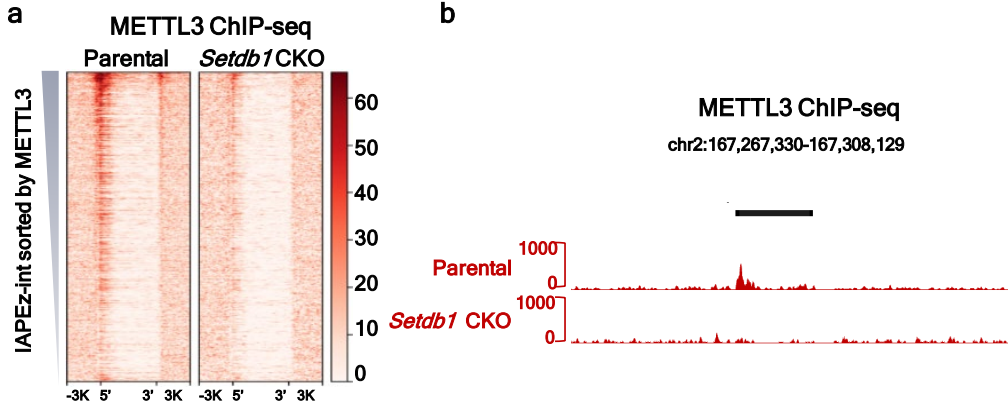

d

METTL3 ChIP-seq
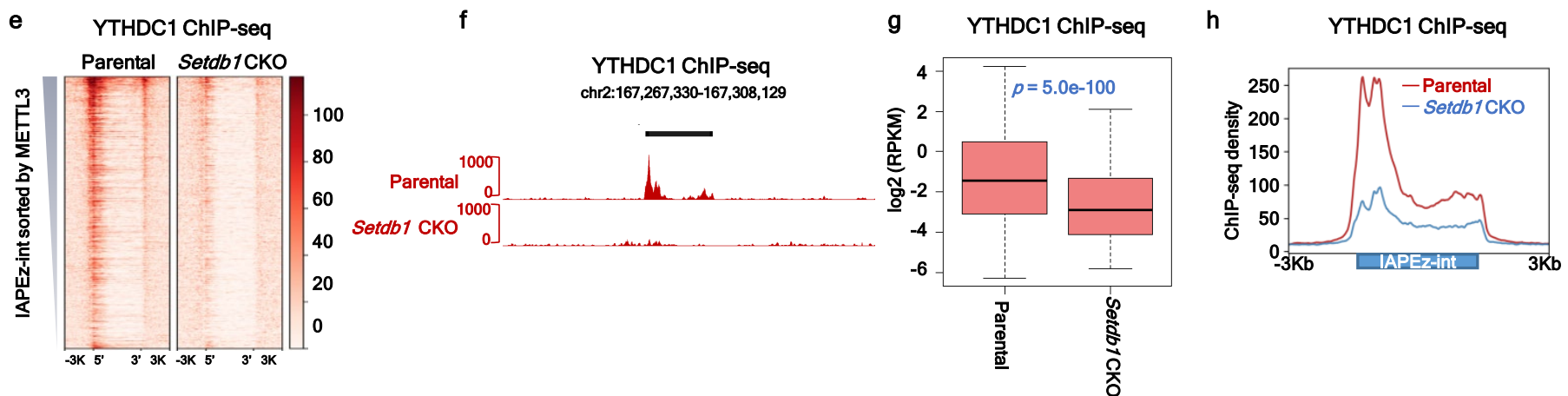

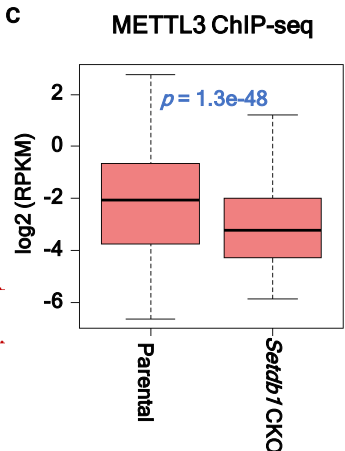

g YTHDC1ChIP-seq h

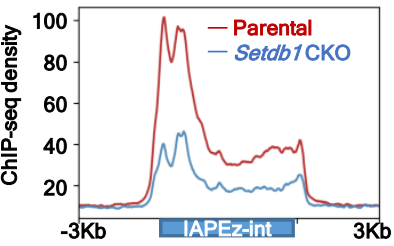


Extended Data Fig. 10. RNA dependent heterochromatin formation models

a

Mus Musculus (IAPEz elements)

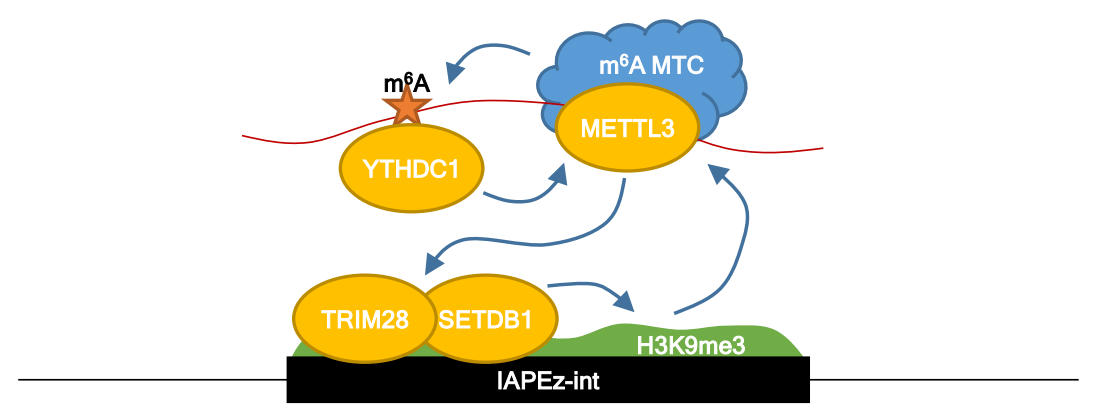

b

S. Pombe (Centromere)

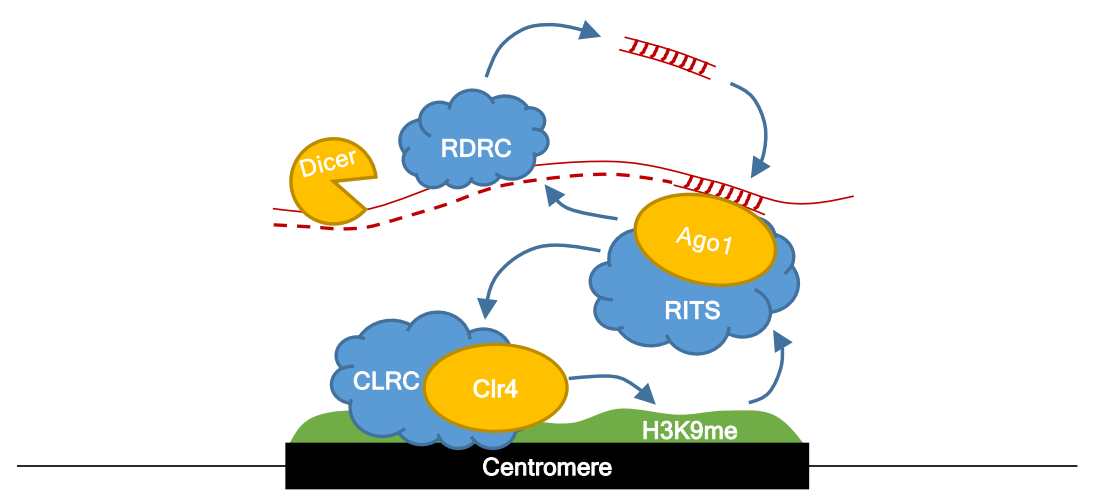

S. Pombe(DSR genes)

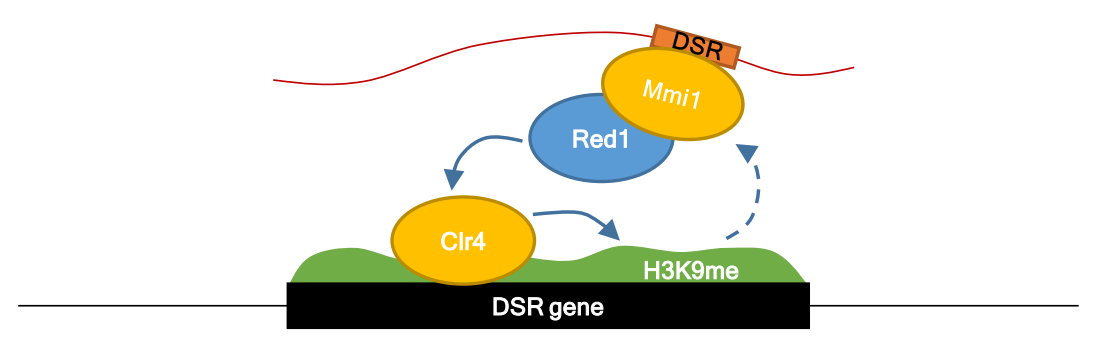

